\title{
Improving biological production of poly(3-hydroxybutyrate- co-3-hydroxyvalerate) (PHBV) co-polymer: a critical review
}

\author{
Grazia Policastro (i) - Antonio Panico • Massimiliano Fabbricino
}

Received: 23 January 2021 / Accepted: 2 April 2021/Published online: 13 April 2021

(C) The Author(s) 2021

\begin{abstract}
Although poly(3-hydroxybutyrate-co-3hydroxyvalerate) (PHBV) is the most promising biopolymer for petroleum-based plastics replacement, the low processes productivity as well as the high sale price represent a major barrier for its widespread usage. The present work examines comparatively the existing methods to enhance the yield of the PHBV copolymer biologically produced and/or reduce their costs. The study is addressed to researchers working on the development of new biological production methods and/or the improvement of those currently used. At this aim, the authors have considered the analysis of some crucial aspects related to substrates and microorganism's choice. The production strategies have been individuated, presented and discussed, either based on a single aspect (type of substrate or microorganism) or based on combined aspects (type of substrate and microorganism). Process operating conditions have been discussed as well. The analysis
\end{abstract}

G. Policastro $(\varangle) \cdot$ M. Fabbricino

Department of Civil, Architectural and Environmental

Engineering, University of Naples "Federico II", via

Claudio 21, 80125 Naples, Italy

e-mail: grazia.policastro@unina.it

M. Fabbricino

e-mail: massimiliano.fabbricino@unina.it

\section{A. Panico}

Department of Engineering, University of Campania

"Luigi Vanvitelli", via Roma 29, 81031 Aversa, Italy

e-mail: antonio.panico1@unicampania.it indicates that the addition of $3 \mathrm{HV}$ precursors is capable to dramatically enhance the hydroxyvalerate fraction in the produced biopolymers. On the other hand, due to the high costs of the $3 \mathrm{HV}$ precursors, the utilization of wild bacterial species capable to produce the hydroxyvalerate fraction from unrelated carbon sources (i.e. no $3 \mathrm{HV}$ precursors) also can be considered a valuable strategy for costs reduction. Moreover, metabolic engineering techniques can be successfully used to promote $3 \mathrm{HV}$ precursors-independent biosynthesis pathways and enhance the process productivity. The use of mixed cultures or extremophile bacteria avoids the need of sterile working conditions, and therefore favours the process scale-up. The utilization of the organic waste as substrate plays a key role for a sharp reduction of production costs. Finally, the selection of the most suitable substrate-microorganism combination cannot be separated by the adoption of an appropriate choice of reactor configuration and abiotic factors. 


\section{Graphic abstract}

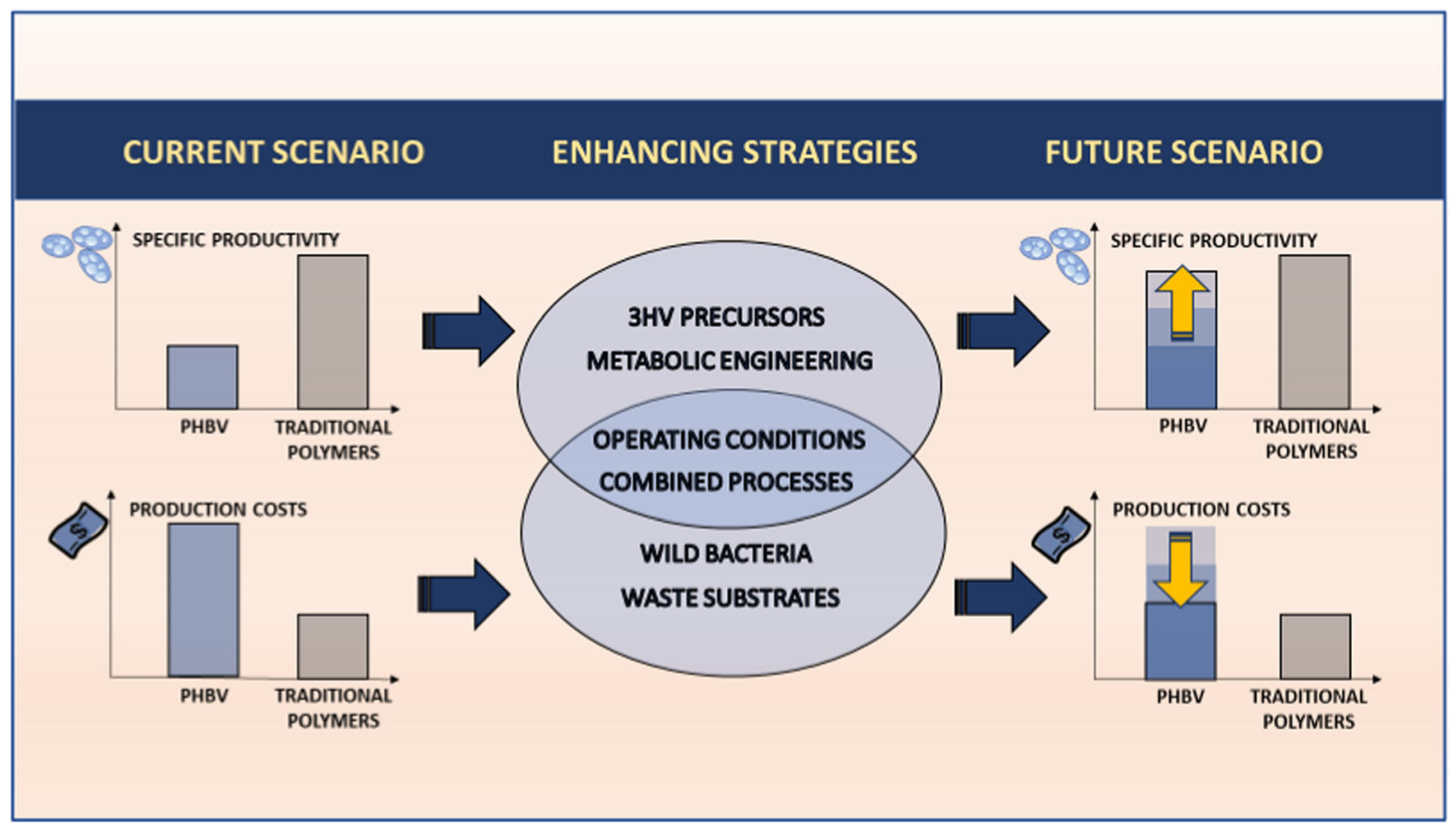

Keywords $3 \mathrm{HV}$ precursors $\cdot$ Biorefinery $\cdot$ Metabolic engineered microorganisms $\cdot$ High performance biomaterials $\cdot$ PHBV

\section{Introduction}

The discovery of polyhydroxyalkanoates (PHAs) dates back to 1888 , when Martinus W. Beijerinck, one of the cofounders of the environmental microbiology, observed, for the first time, PHAs granules in microorganisms' cytoplasm (Khanna and Srivastava 2005). Over the next 80 years, scientists kept studying the microbial synthesis of various PHAs as an academic concern (Raza et al. 2019). Only in the last few decades, due to the need of finding biodegradable materials capable to replace conventional plastics, the studies on PHAs production have assumed a primary interest. Indeed, from an environmental perspective, PHAs are the most suitable biopolymers for the production of biodegradable plastic materials (Li et al. 2016). Such conclusion has been deduced through different Life Cicle Assessment (LCA) studies, performed on various types of bioplastics as well as conventional plastics. Results from these analyses showed that, in terms of energy demand and greenhouse gases emissions, production and use of all bioplastics is more advantageous than conventional plastics. Conversely, bioplastics, such as those based on starch and corn, have a strong impact on the environment due to soil acidification and surface waters eutrophication because of fertilizers and chemicals used to cultivate the raw materials (Gironi and Piemonte 2011). Among the different types of bioplastics, PHAs have the advantage to be produced from waste materials (Policastro et al. 2020), thus avoiding the occurrence of the above mentioned negative effects on the environment.

Currently, researchers are focusing their attention on the enhancement of PHAs properties to promote their use in various applications (Zheng et al. 2020). It has been demonstrated, in fact, that different species of microorganisms are capable to incorporate hydroxyvalerate $(3 \mathrm{HV})$ units into the PHB molecule, which is the most studied compound belonging to the family 
of PHAs (Byrom 1992; Zuñiga et al. 2013). The result is a co-polymer, the poly(3-hydroxybutyrate-co-3hydroxyvalerate), widely known with the acronym of PHBV, that owns superior thermal and mechanical properties compared to PHB and all other PHAs. Due to enhanced physical and chemical characteristics, such as better mechanical flexibility and strength, shorter chain packing and lower toughness, PHBV is gaining attention of many researchers (Tebaldi et al. 2019). Compared to other PHAs, PHBV has become the most promising biopolymer to replace petroleumbased plastics in a wide range of applications (e.g. tissue engineering, biomaterial applications, disposable as well high mechanical resistance objects production) (Rivera-Briso and Serrano-Aroca 2018). Moreover, due to its superior characteristics compared to other PHAs and other biopolymers, PHBV is particularly attractive for biomedical applications as well (Tebaldi et al. 2019).

However, the high sale price of PHBV still represents a major barrier to its widespread diffusion (Yu et al. 2006). On the base of techno-economic analysis, the PHBV production costs strongly depend on specific process conditions (Bhattacharyya et al. 2015). Therefore, it is necessary to address further efforts to enhance the production process efficiency as well as reduce the final cost of the product. A comprehensive review of the existing methods used to optimize the PHBV production is certainly relevant as starting point to better address future investigations. An updated and critical analysis of such strategies, object of the present work, is currently absent in the scientific literature. In particular the paper contains the analysis of strategies concerning the enhancement of the productivity as well as the reduction of production costs, individuated on the basis of the available techno-economic analysis, thus contributing to reduce the competitiveness gap between PHBV and traditional plastics. More in details, the paper presents a comprehensive review of a massive number of published studies on strategies focused on improving the biological production of the co-polymer PHBV. The relevant information on the processes used to produce PHBV have been pointed out and compared. The peculiarities and the effectiveness of the adopted microbial species and substrates have been analysed. The efficiency of microorganisms, substrates and the microorganism-substrate combination have been assessed in terms of PHBV accumulation and hydroxyvalerate (3HV) monomer fraction. The most significant production strategies have been critically presented and discussed, highlighting, for each of them, the advantages as well as the disadvantages, in order to guide readers towards a reasoned decision that might be suitable for their specific scope.

To facilitate the analysis, the reviewed studies are divided into two main groups. The first group includes all strategies based on microorganisms' selection, either the use of wild microorganisms or those metabolic engineered. The second group, instead, includes all strategies based on substrate selection and/ or substrate pre-treatment/modifications (e.g. $3 \mathrm{HV}$ precursors co-substrates addition). Operating process conditions and combinations of different strategies have been discussed as well. A final discussion on all presented strategies is critically conducted, with the aim of focusing those most performing. The study is, therefore, of great concern for researchers interested in developing new methods to produce PHBV and/or improve those currently used, with the aim of achieving optimal operating conditions, effective and efficient enough to promote an economically convenient full-scale production of PHBV.

\section{PHBV biosynthesis processes, characteristics and applications}

PHBV, also indicated as PHBHV or P(3HB-co-3HV), is a thermoplastic bio-polyester that structurally originates from the insertion of a $3 \mathrm{HV}$ unit into the PHB polymer structure (Fig. 1).

PHBV, as all other PHAs, is the product of biosynthesis of a wide variety of both gram-positive and gram-negative bacteria (Rivera-Briso and Serrano-Aroca 2018). Among wild microbes, Ralstonia eutropha, also known as Cupriavidus necator or Alcaligenes eutrophus (Kim et al. 1994; Chung et al. 2001; Lee et al. 2008; $\mathrm{Ng}$ et al. 2011; Grousseau et al. 2014) has been the most used specie. This bacterial

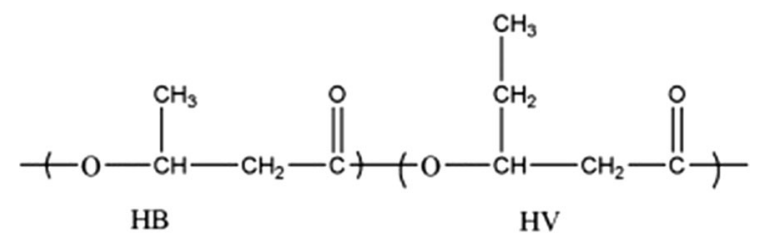

Fig. 1 PHBV chemical structure 
strain can accumulate high PHBV amounts under unbalanced growth conditions (i.e. lack of nitrogen, phosphorus or sulfur). Recently, the archea Haloferax Mediterranei, an extremely halophilic microrganism, has gained a greater attention, due to its faster growth, its high PHBV productivity and its capacity of producing high quality products (Hou et al. 2013). Different species of Bacillus, Methilobacterium, Pseudomonas and Rhodospirillacee have been also tested on various substrates (Ueda et al. 1992; Smith et al. 2008; Zuñiga et al. 2013; Martla et al. 2018; Balakrishna Pillai et al. 2020). Finally, even though less studied if compared to the previous mentioned microorganisms, Alcaligenes, Comamonas, Halomonas and Rhodococcus have been found to be capable to produce PHBV (Choi et al., 2003; Kulkarni et al., 2010; Williams et al., 1994; Zakaria et al., 2013). Microorganisms store PHAs in form of intracellular granules, with the aim of using them as energy reserve. PHAs accumulation is a strategy of microorganisms to increase their chance of survival under adverse environmental conditions (Policastro et al. 2020). Specifically, microorganisms can follow the metabolic pathway that results in PHAs production whenever one or more of the following specific conditions occur (Steinbüchel and Lütke-Eversloh 2003): (1) environmental signals, such as nutrient starvation, that leads to the activation of the PHA-gene expression; (2) presence of specific metabolic intermediates or cell components that activate the PHA synthetic enzymes; (3) enrichment of the required intermediates for PHA synthesis due to the inhibition of competing metabolic pathways.

Metabolic pathways promoting PHBV biosynthesis principally depend on the characteristics of the bacterial strain. The majority of PHBV accumulating bacterial species store the biopolymer under nutrient (e.g. nitrogen, phosphorous or sulphur) starvation with excess of carbon source (Rivera-Briso and SerranoAroca 2018).

Figure 2 shows the simplified PHBV production pathway from glucose and propionic acid.

As reported in Fig. 2, the PHBV biosynthesis is catalysed by two key enzymes: the 3-hydroxybutylCoA (3HBCoA) and the 3-hydroxyvaleryl-CoA (3HVCoA). While the $3 \mathrm{HBCoA}$ can be obtained from a wide range of substrates by a large variety of bacteria, the majority of PHBV accumulating bacteria

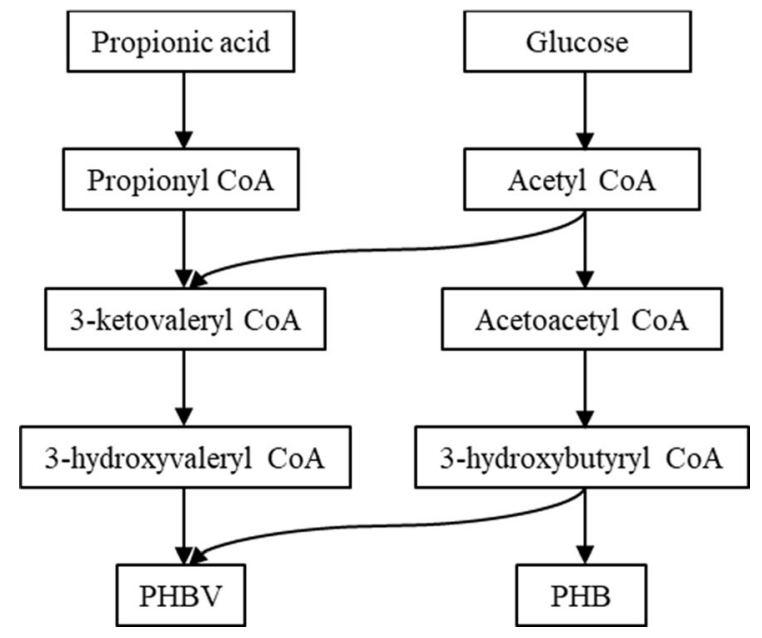

Fig. 2 PHB and PHBV production pathways

requires the presence of $3 \mathrm{HV}$ precursors (e.g. valerate and propionate) to synthetize the $3 \mathrm{HVCoA}$.

Concerning the properties of PHBV, they have been extensively reviewed by Laycock et al. (2014). To sum them up, the $3 \mathrm{HV}$ fraction determines the defection of the PHB lamellae crystals, thus leading to the disruption of the PHB crystallinity (Laycock et al. 2014). The result is a more flexible structure of the biocopolymer compared to the structure of polyhydroxybutirate-PHB. Such an improved flexibility is responsible for a general enhancement of all its mechanical properties (Vu et al. 2020). A comparison of the main properties among PHBV, PHB and the widest diffused traditional plastic (low density polyethylene) are reported in Table 1.

PHBV is tougher and more elastic than PHB. The lower melting point of PHBV makes it easier and less expensive to be processed if compared to PHB. Moreover, as it can be easily noticed from Table 1, PHBV owns properties more similar to the traditional low density polypropylene than PHB (Strong et al. 2016; Poltronieri and Kumar 2017). Compared to conventional plastic materials, PHBV shows similar physical and mechanical properties with the advantage of being totally biodegradable and biocompatible with a wide variety of cells (Bugnicourt et al. 2014). In addition, it is non-toxic, and resistant to ultraviolet radiation as well as to several alcohols, fats, and oils (Bugnicourt et al. 2014). Finally, at the end of its life cycle, PHBV based polymers can be conveniently valorised as renewable energy and/or material source. 
Table 1 PHBV, PHB and low density polypropylene properties (Adapted from Strong et al. (2016))

\begin{tabular}{lllllr}
\hline Polymer & $\begin{array}{l}\text { Melting } \\
\text { point }\left({ }^{\circ} \mathrm{C}\right)\end{array}$ & $\begin{array}{l}\text { Glass-transition } \\
\text { temperature }\left({ }^{\circ} \mathrm{C}\right)\end{array}$ & $\begin{array}{l}\text { Young's } \\
\text { modulus (GPa) }\end{array}$ & $\begin{array}{l}\text { Tensile } \\
\text { strength (MPa) }\end{array}$ & $\begin{array}{l}\text { Elongation } \\
\text { to break }(\%)\end{array}$ \\
\hline PHB & 180 & 4 & 3.5 & 40 & 5 \\
P(3HB-co-20 mol\%£HV) & 145 & -1 & 0.8 & 20 & 50 \\
Low density polypropylene & 130 & -30 & 0.2 & 10 & 620 \\
\hline
\end{tabular}

At this aim, both a mechanical recycling, conducted through extrusion, and a chemical recycling, conducted thorough pyrolysis, result to be effective (Morikawa and Marchessault 1981; Zembouai et al. 2014). Alternatively, the bio-copolymer can be used as substrate for biofuels production, through anaerobic digestion or dark fermentation process $(\mathrm{Vu}$ et al. 2020).

Currently, PHBV is the most commonly used copolymer to prepare high-performance biopolymers (Ashori et al. 2019). Without further modifications, PHBV can be used for various applications (e.g. controlled release of drugs, medical implants and repairs, packaging, orthopedic devices, disposable objects, etc....). Moreover, combining the copolymer with other natural materials (e.g. fibres, other polymers, carbon nanomaterials) allows producing a wide spectrum of biomaterials with different structures and enhanced mechanical properties (Rivera-Briso and Serrano-Aroca 2018). The physical and mechanical properties of PHBV greatly depend on the $3 \mathrm{HV}$ content in the copolymer. Indeed, increasing $3 \mathrm{HV}$ content enhances the biodegradability and reduces the crystallinity and melting point of the bio-copolymer. Therefore, according to the required application, a suitable $3 \mathrm{HV}$ fraction should be reached by modifying the operational conditions of the process (Rivera-Briso and Serrano-Aroca 2018).

\section{Techno-economic analysis and production strategies}

Currently, the production costs of PHBV have been approximately estimated to range between 1.50 and 10 $\$ / \mathrm{Kg}_{\mathrm{PHA}}$, depending on the production plant location and, principally, on the adopted operating conditions (Garcia et al. 2011; Hermann-Krauss et al. 2013; Bhattacharyya et al. 2015).

The analysis of the PHAs production costs is of primary concern for the selection of strategies aimed at enhancing the process performance. Usually, a technoeconomic analysis considers fixed capital and annual operating costs. Fixed capital costs include both direct and indirect plant costs and other costs such as contractor's fee and contingency. The annual operating costs regards the management of the direct fixed capital-dependent items as well as labor-dependent items, the administration and overhead expenses, the raw materials purchase, the utilities and waste treatment/disposal costs (Choi and Lee 1997). All these costs are strongly dependent on production factors.

First of all, equipment-related costs considerably increase when the productivity decreases. Indeed, for the production of the same amount of PHA per year, the process with lower productivity $\left(\mathrm{g}_{\mathrm{PHA}} / \mathrm{L} / \mathrm{h}\right)$ requires larger both reactor and equipment size (Choi and Lee 1999b). Moreover, the PHA content and the PHA yield in terms of used carbon source affect the biopolymer recovery process efficiency. Indeed, higher PHA content requires less amount of digesting agents to separate granules from cells. For instance, Choi and Lee (1999b) estimated a recovery cost of $4.8 \$ / \mathrm{kg}_{\text {PHA }}$ when the PHA content in cells was $50 \%$ $(\mathrm{m} / \mathrm{m})$. Nevertheless, the cost decreased to $0.92 \$ /$ $\mathrm{kg}_{\text {PHA }}$ with $88 \%$ PHA content $(\mathrm{m} / \mathrm{m})$ in cells. In addition, low PHA yields cause a large amount of carbon substrate to be wasted. Koller et al. (2007a) performed the techno-economic analysis in producing PHAs by comparing different species of microorganisms. They estimated that the polymers produced by $P$. hydrogenovora and $H$. mediterranei could be manufactured at the prices of 10.5 and 2.82 euros per $\mathrm{Kg}_{\text {PHA }}$, respectively. The lowest costs achieved using H. Mediterranei were due to the higher PHBV concentration and productivity. In addition, as $H$. mediterranei do not require sterile conditions, energy demand to set such conditions was null.

The cost of the carbon source also contributes significantly to the overall production costs. Chanprateep (2010) calculated that raw material accounts for $30-40 \%$ of the total costs. Also, Choi and Lee (1999b) 
estimated that the cost of the carbon source was $38 \%$ of the total operating costs when the production amounts to 100000 tonnes/year. Further details were provided by Choi et al. (2010), who performed a sensitivity analysis: the PHA production costs depended significantly on changes in feedstock price. When the substrate cost was reduced from 55 to $22 \$$ / $\mathrm{Mg}$, the PHA production costs were reduced to $0.05 \$ /$ $\mathrm{kg}_{\text {PHA }}$. When the substrate cost increased to $88 \$ / \mathrm{Mg}$, the PHA production cost increased by $115 \%$. Similarly, Bhattacharyya et al. (2015) observed in their study that the raw material costs accounted only for $20 \%$ the costs estimated by Garcia et al. (2011) for the PHBV production in similar operating conditions. The carbon source required in the process proposed by Garcia et al. (2011) costed $0.22 \$ / \mathrm{kg}_{\text {PHA }}$. This value significantly affected the final production costs. On the other hand, the stillage used as substrate by Bhattacharyya et al. (2015) had no cost, because it was a waste. Moreover, such waste already contained a propionate concentration of $0.65 \mathrm{~g} / \mathrm{L}$, therefore PHBV production did not require additional costs due to $3 \mathrm{HV}$ precursor supply. Indeed, for most practical uses of $\mathrm{PHBV}$, the $3 \mathrm{HV}$ fraction of the biopolymer should be at least in the range of $10-20 \%(\mathrm{~m} / \mathrm{m})$ (HermannKrauss et al. 2013) and usually precursors (e.g. propionate and valerate) are required to be added to achieve such $3 \mathrm{HV}$ fraction (Hermann-Krauss et al. 2013). On the other hand, the use of waste substrates could require additional high equipment costs due to pre-treatments, as reported by Garcia et al. (2011), who performed extrusion of rice/wheat bran and corn starch prior to starting the PHBV production phase.

Concerning the operational conditions costs, oxygen supply to maintain aerobic conditions can be potentially costly (Wegen et al. 1998). Indeed, prevention of oxygen limitation generally requires a pressurized vessel, high mixing energy and oxygenenriched air feeds. Therefore, the production costs increase significantly (Choi and Lee 1999b). Moreover, Akiyama et al. (2003) proved that the effect of aeration rate was more significant on costs compared to the effect of temperature.

The combination of different processes that can generate both energy and PHAs could significantly reduce the global costs. For instance, Choi et al. (2010) performed the sensitivity analysis concerning costs of combined processes producing PHAs and hydrogen. The production costs of PHAs was significantly influenced by changes in the hydrogen market price: reducing the hydrogen market price from 2.0 to $0.8 \$$ / $\mathrm{kg}$, the PHA production costs increased by $191 \%$ up to $6.46 \$ / \mathrm{kg}_{\text {PHA }}$. A higher hydrogen market price of $3.2 \$ /$ $\mathrm{kg}$ resulted in a decrease of the PHA production costs down to $3.15 \$ / \mathrm{kg}_{\mathrm{PHA}}$. Similar result could be reached by increasing the hydrogen productivity.

Based on economic analysis and the techniques used to produce PHBV in the published papers, different production strategies have been considered to be promising.

The addition of precursors has been widely used and has been therefore individuated as a strategy aimed at PHBV productivity enhancement and 3HV fraction control. Alternatively, it has been successfully tested the utilization of bacterial species that do not require precursors. The use of specific bacterial strains has also been supported when this practice is capable to avoid costs of sterilization and/or ensures a higher productivity. Finally, metabolic engineering techniques have been tested and proposed, obtaining interesting results: Ralstonia eutropha, Haloferax Mediterranei and Alcaliges species have been modified to enhance their productivity (Choi et al. 2003; Yoon et al. 1995; Zhao et al. 2013). Moreover, other species such as Escherichia coli, Halomonas, Aeromonas and Salmonella enterica, which do not produce biopolymers, have been engineered to produce PHBV (Aldor et al. 2002; Wu et al. 2012; Miscevic et al. 2019; Shi et al. 2020).

As far as concern the choice of the most appropriate substrate to be used, both primary and waste substrates have been tested. The majority of studies have been carried out using pure substrates (e.g. glucose, glycerol, starch, methane, oils and volatile fatty acids) with high nutritional value and/or prize. However, in PHAs production processes less than half of the carbon source is directed towards PHAs accumulation (Hermann-Krauss et al. 2013), because it is used for the intracellular respiration and the production of other metabolites. Consequently, carbon supply largely affects PHAs production costs. Clearly, the utilization of waste material as feedstock is a strategy of main importance as it considerably reduces both costs related to substrates supply and waste disposal. Therefore, over the last few years, researchers have tested several waste materials to verify their PHBV production efficiency ( $\mathrm{Du}$ and $\mathrm{Yu}$ 2002; Bhattacharyya et al. 2012; Pais et al. 2016). Due to the 
complexity of real substrates, in most cases, the use of waste material requires the adoption of appropriate pre-treatment techniques. The aim of pre-treatments is to facilitate the degradation process and/or avoid the possible inhibition of microorganisms' activity. On the other hand, pre-treatments also require additional costs. Table 2 reports a summary of the most relevant studies that have developed and tested the most promising strategies reported in the present work. All the strategies have been presented and discussed in the following sections.

\section{Microorganisms as production strategy}

As previously said, a wide range of both gram-positive and gram-negative bacteria have been found to be capable to produce PHBV.

To enhance the process productivity and contextually reduce the costs, the choice of microorganisms represents a crucial aspect, and therefore it can be considered as a production strategy. For this reason, in this section are reviewed and compared results of research studies, which have used different bacterial species, including those that have tested metabolic engineering techniques.

\subsection{Wild species}

According to the Table 2, the gram-negative bacterium Ralstonia eutropha is one of the most frequently tested microorganisms. Ralstonia eutropha can degrade a wide variety of substrates, including aromatic compounds. Moreover, it is resistant to potential toxic elements (PTE) such as mercury (Pérez-Pantoja et al. 2008). It can utilize wastes containing sugars, alcohols and volatile fatty acids (e.g. acetic, propionic, and butyric acids) as sole carbon and energy source for growing and synthesizing PHB (Cavalheiro et al. 2009; Verlinden et al. 2011). On the other hand, it can produce PHBV only with the addition of precursors containing the $3 \mathrm{HV}$ fraction.

Results obtained using Ralstonia eutropha indicate that this microorganism can accumulate up to $80 \%$ of PHBV in cell dry mass (CDM) when fed with fructose or butyrate (Chung et al. 2001; Grousseau et al. 2014) and more than $90 \%$ when fed with jatropha oil and other plant oils (Lee et al. 2008; Ng et al. 2011). In terms of PHAs concentration, Ralstonia eutropha was found to be capable to produce $117 \mathrm{~g} / \mathrm{L}$ of PHAs using glucose as substrate (Kim et al. 1994), which is the highest production observed in all the reviewed studies.

To maximize the $3 \mathrm{HV}$ fraction in the PHBV polymer, other species of microorganisms have been found to be performant as well. For instance, the bacteria Delftia acidovorans and Caldimnia taiwanensis, fed with valerate, produced PHBV containing a 3HV fraction higher than 90\% (Loo and Sudesh 2007; Sheu et al. 2009).

Rhodospirillaceae, known as purple non sulfur bacteria (PNSB), belong to one of the most versatile family of microrganisms in terms of metabolism. PNSB have attracted increasing attention as they are capable to produce concomitantly hydrogen and PHB from a wide variety of substrates (Policastro et al. 2020). Nevertheless, with the addition of precursors, the specie Rhodobacter Sphaeroides $U 7$ was found to be capable to produce PHBV from volatile fatty acids (VFAs) (Kemavongse et al. 2008).

Obviously, the use of bacteria which do not require costly precursors is advisable to reduce production costs. The absence of precursors results also in important advantages in terms of process management, as precursors have been proved to be harmful to cell growth (Dionisi et al. 2004; Loo and Sudesh 2007), and therefore, to keep their concentration in non-inhibiting thresholds, the process needs a strict control.

Various microorganisms have been studied using the main substrate without any precursors addition. Different species of Bacillus such as Bacillus circulans, Bacillus cereus, Bacillus Flexus and Bacillus OU40T produced PHBV when fed with sugars or industrial wastes (Masood et al. 2012; Phukon et al. 2012; Nagamani and Mahmood 2013; Wagle et al. 2019). Pseudomonas aeruginosa, Yangia ND199 and Halomonas campisalis showed the same interesting capacity (Kulkarni et al. 2010; Van-Thuoc et al. 2015; Ali and Jamil 2017). Nonetheless, in all mentioned studies, PHBV productions were not very high.

Nevertheless, it has been demonstrated that the PNSB Rhodospirillum Rubum synthetizes PHBV from sugars and wastes without the need of precursors supply (Smith et al. 2008; Liu et al. 2019). In particular, in a study from Liu et al. (2019), a $46.5 \%$ $3 \mathrm{HV}$ fraction was observed, which was the highest 


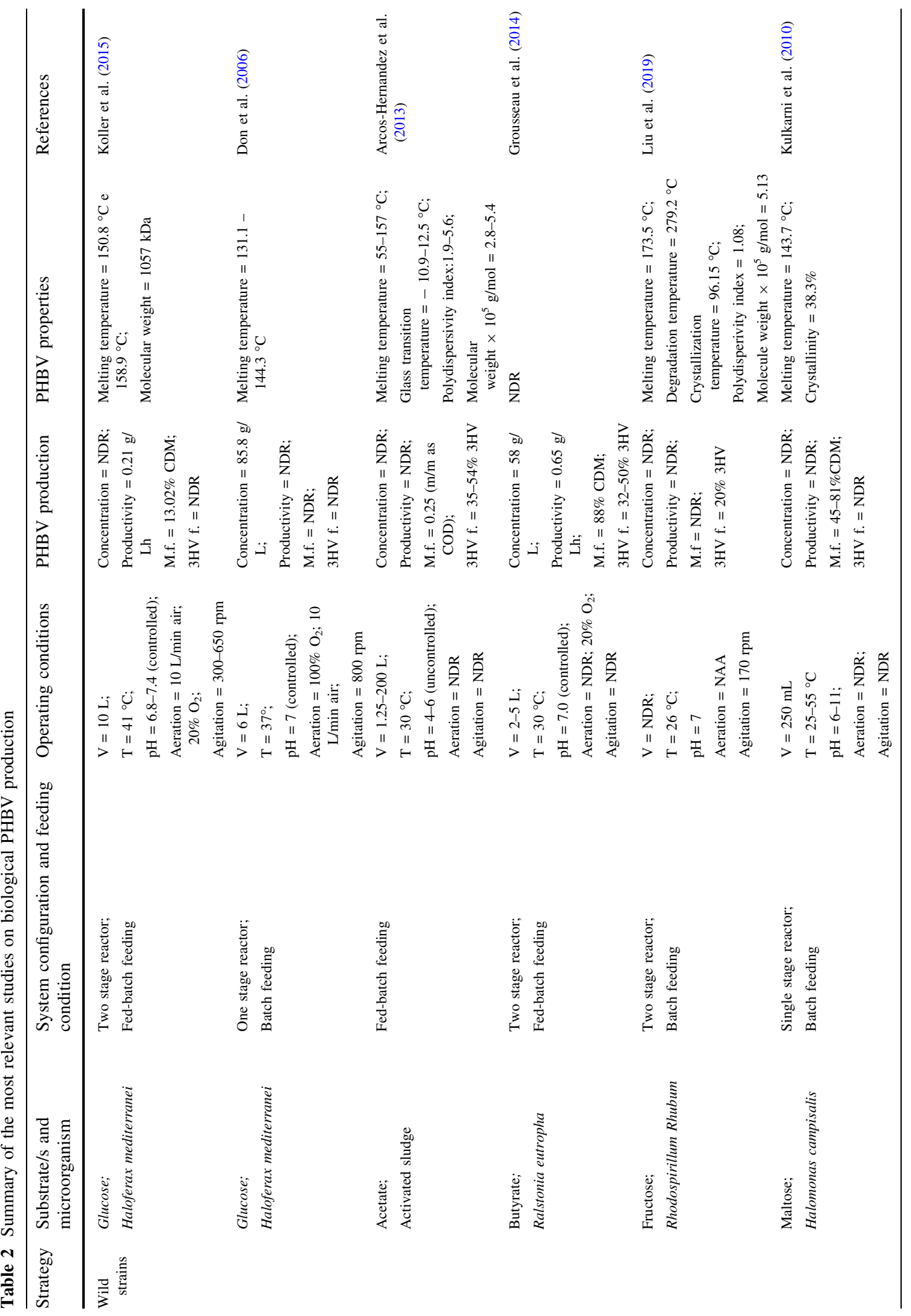




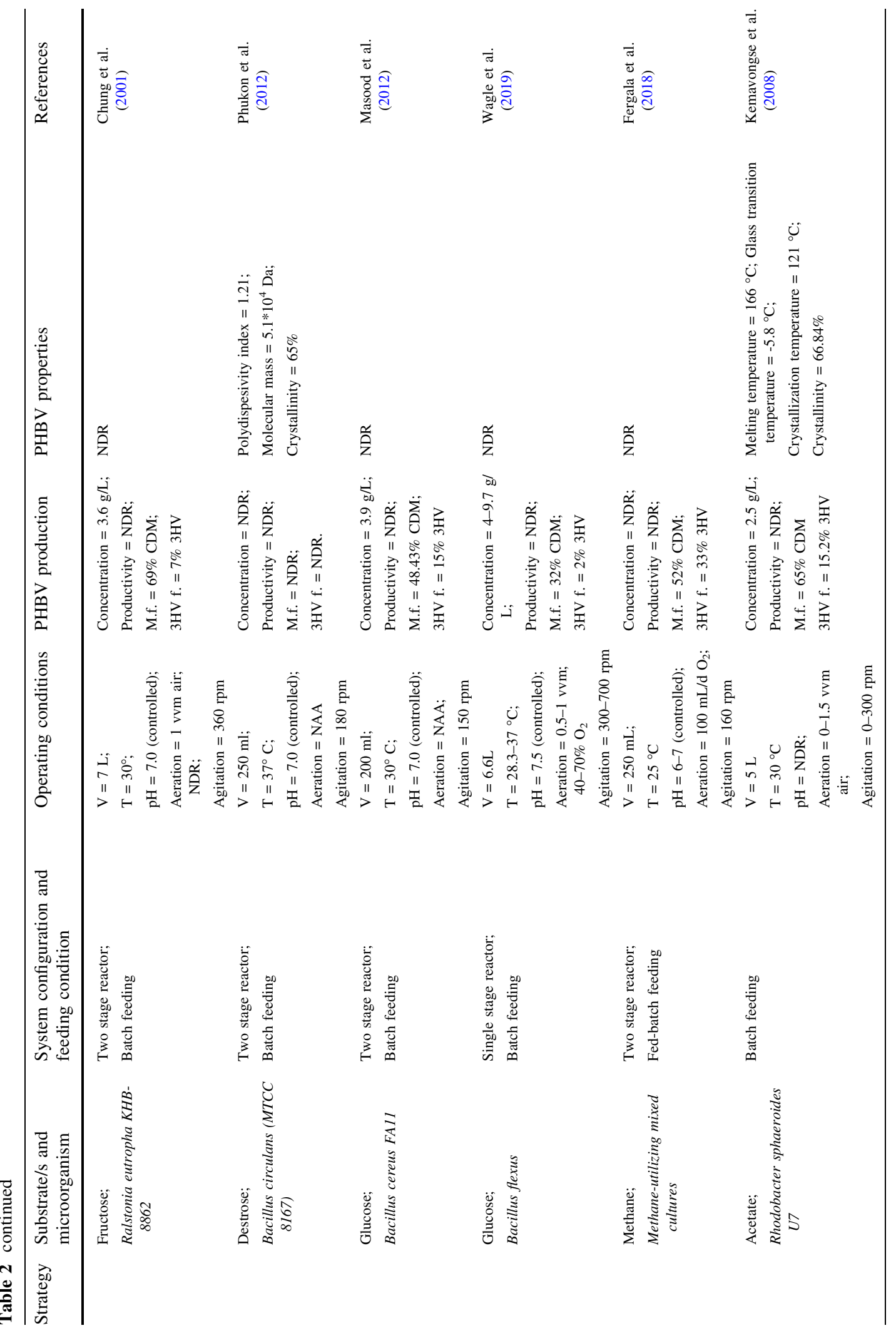




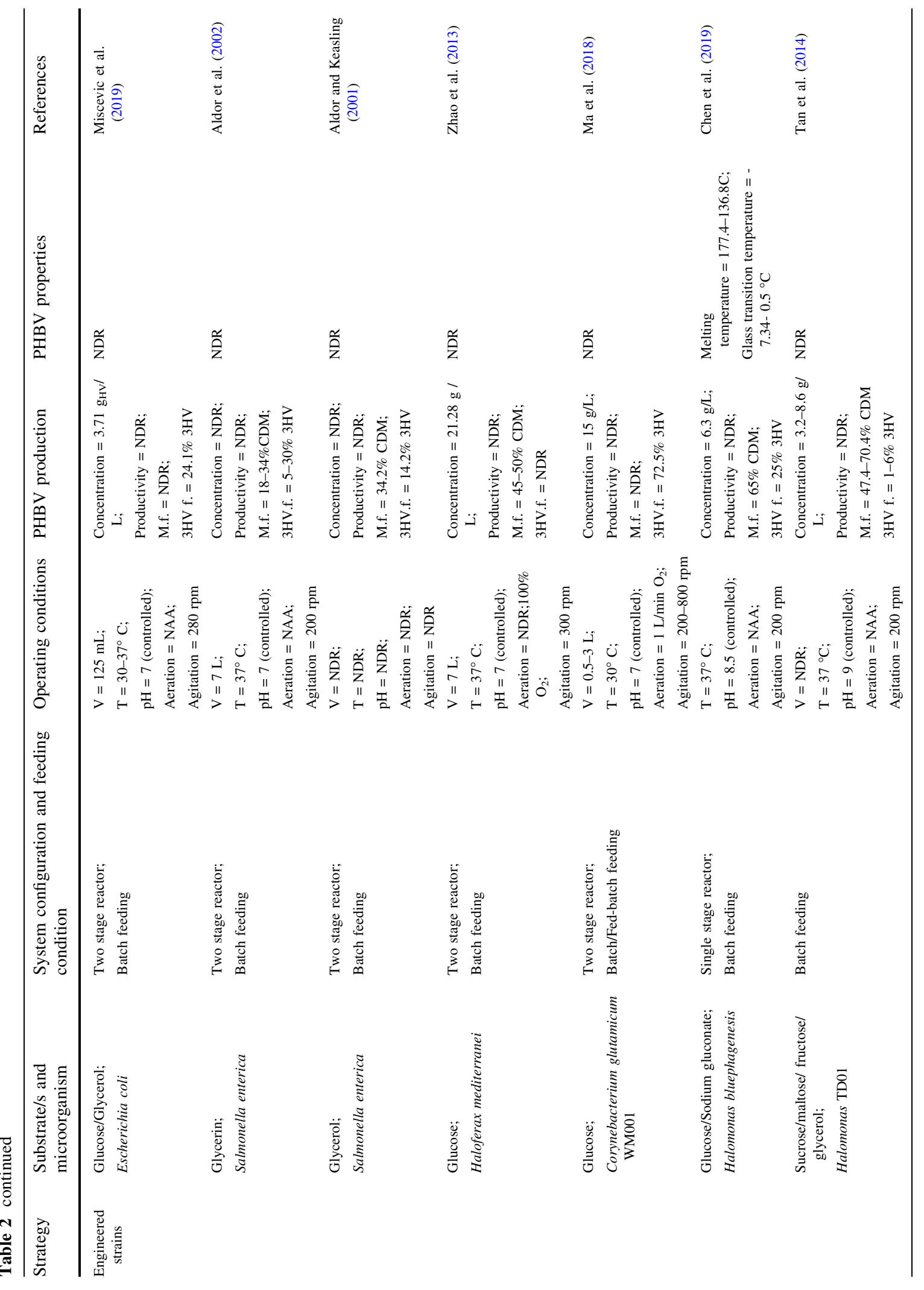




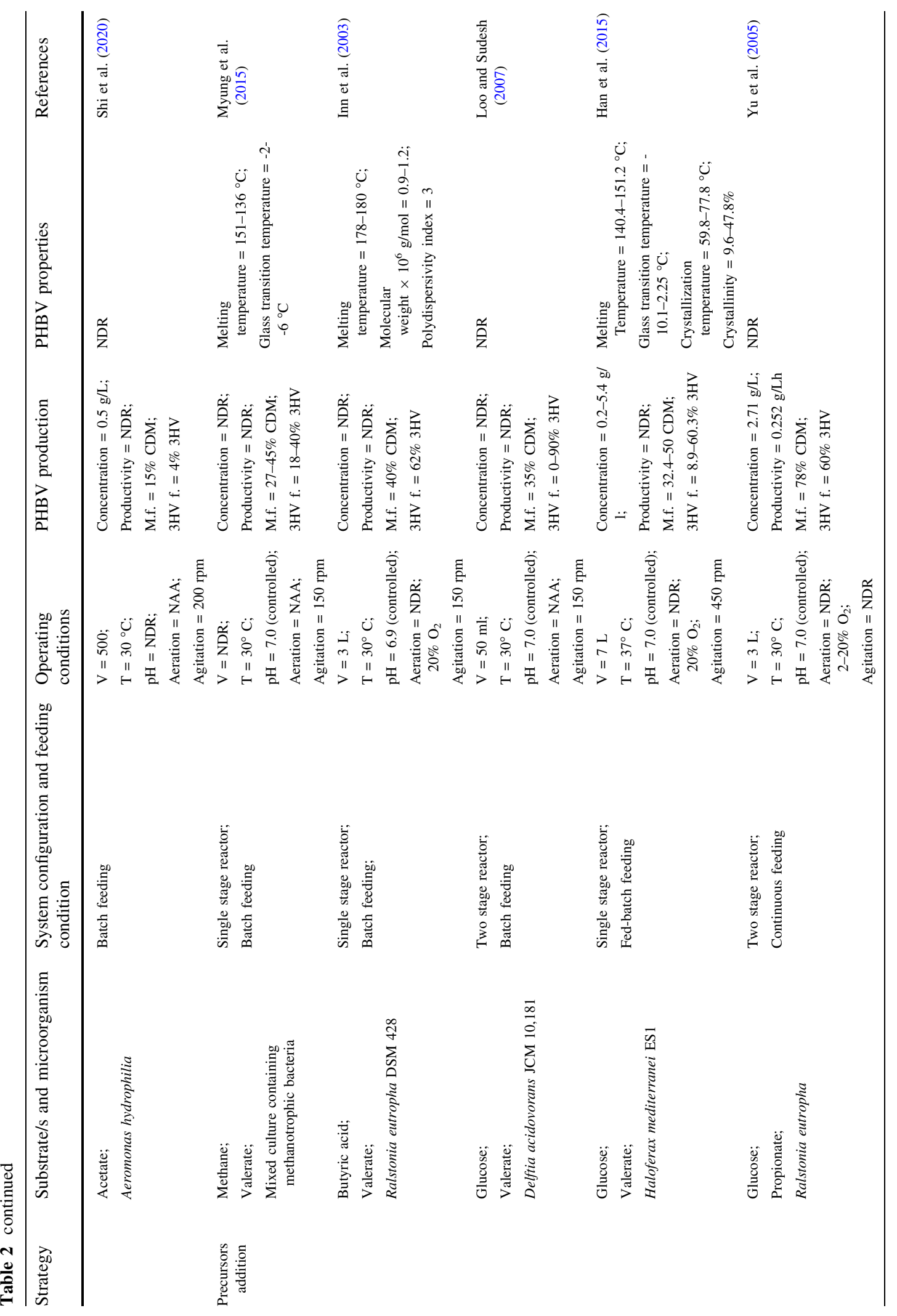




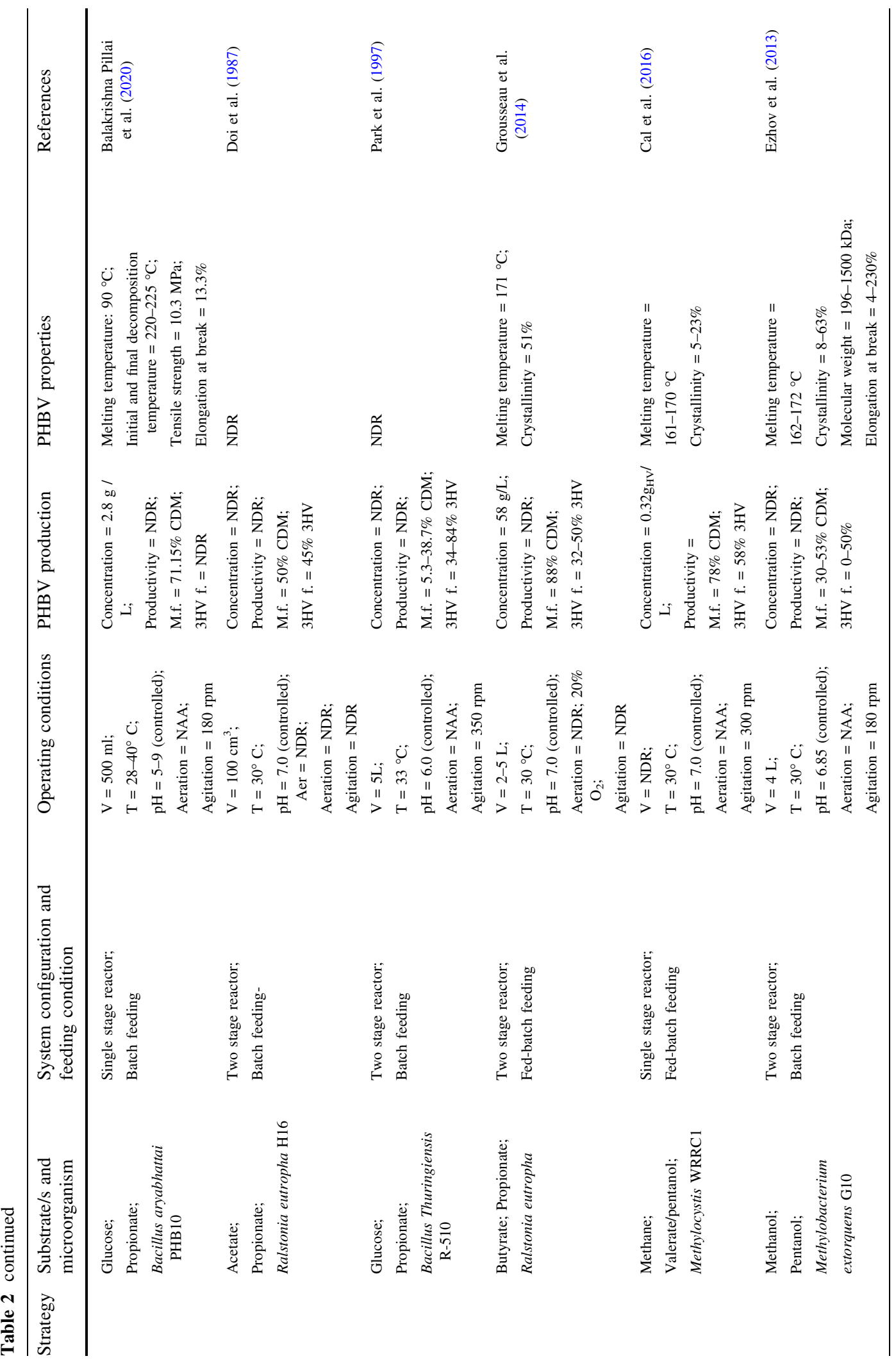




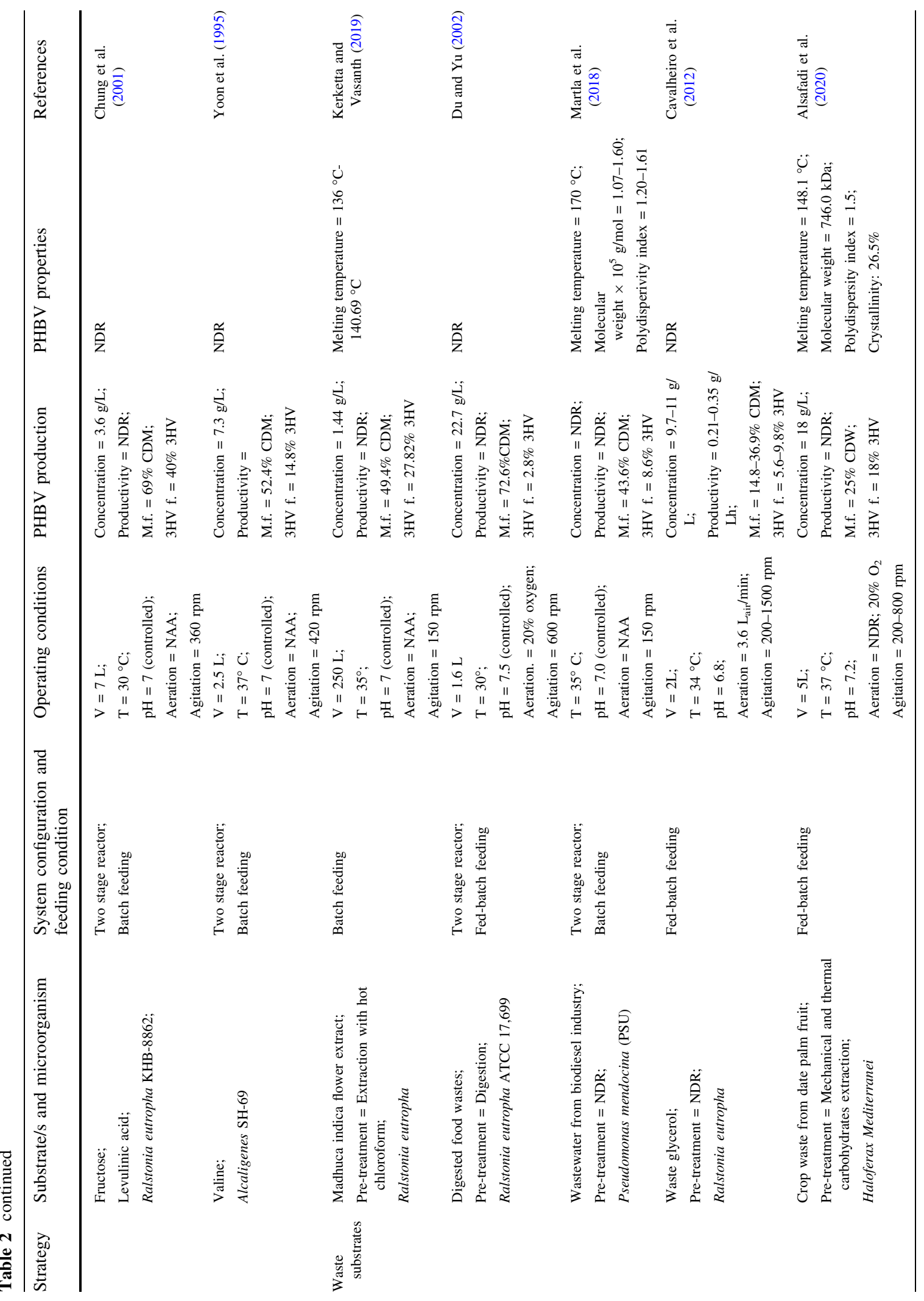




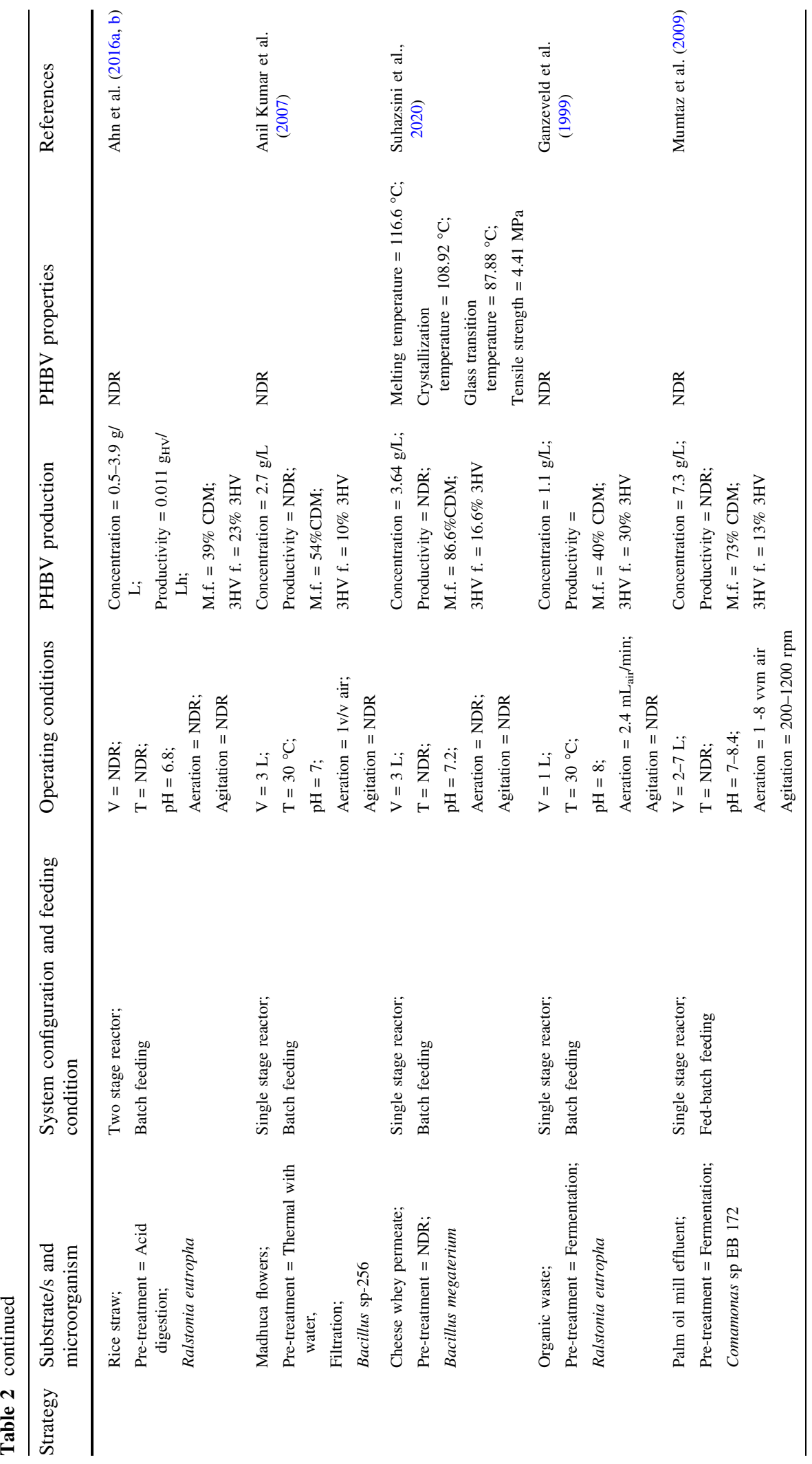




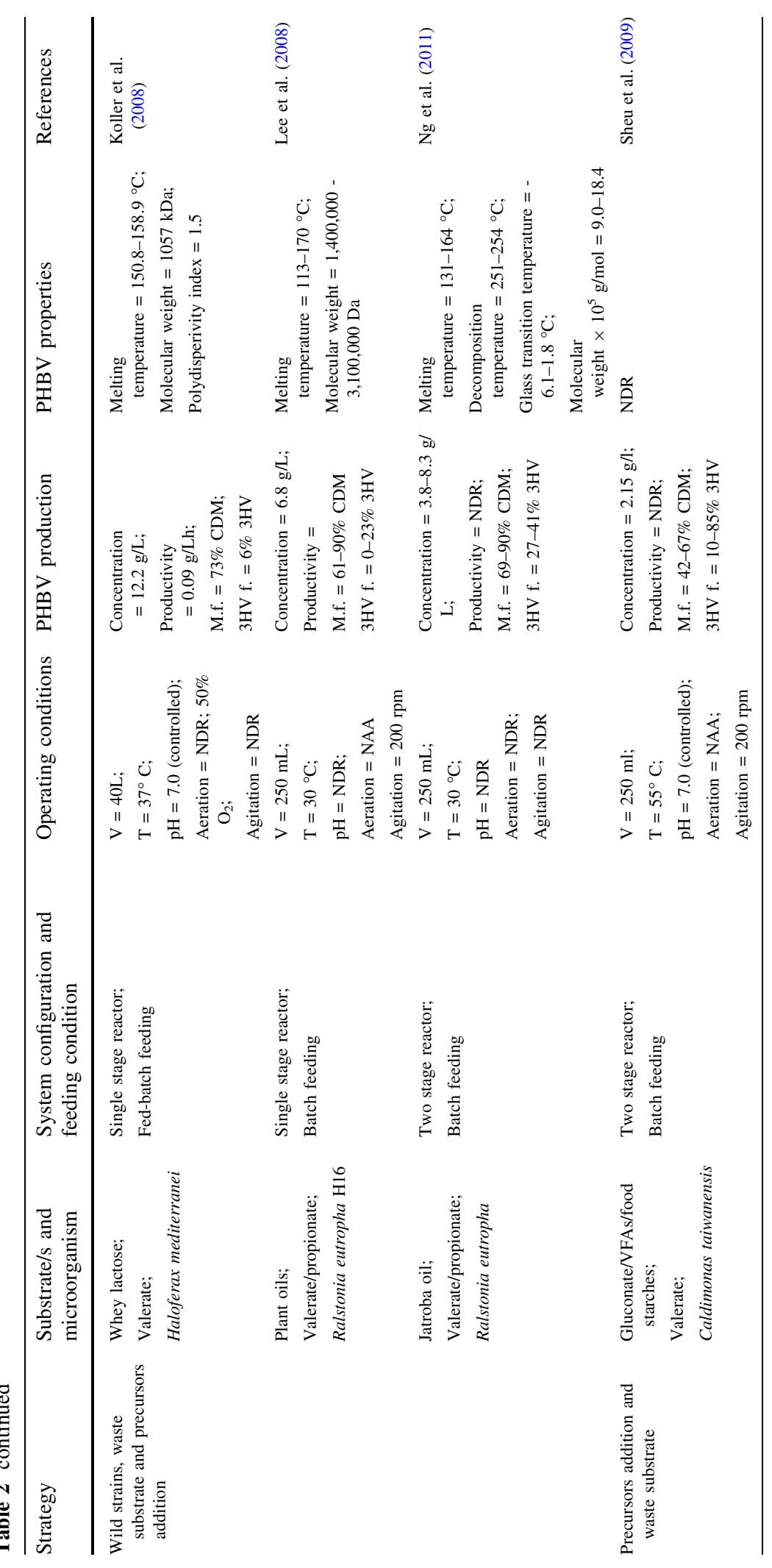




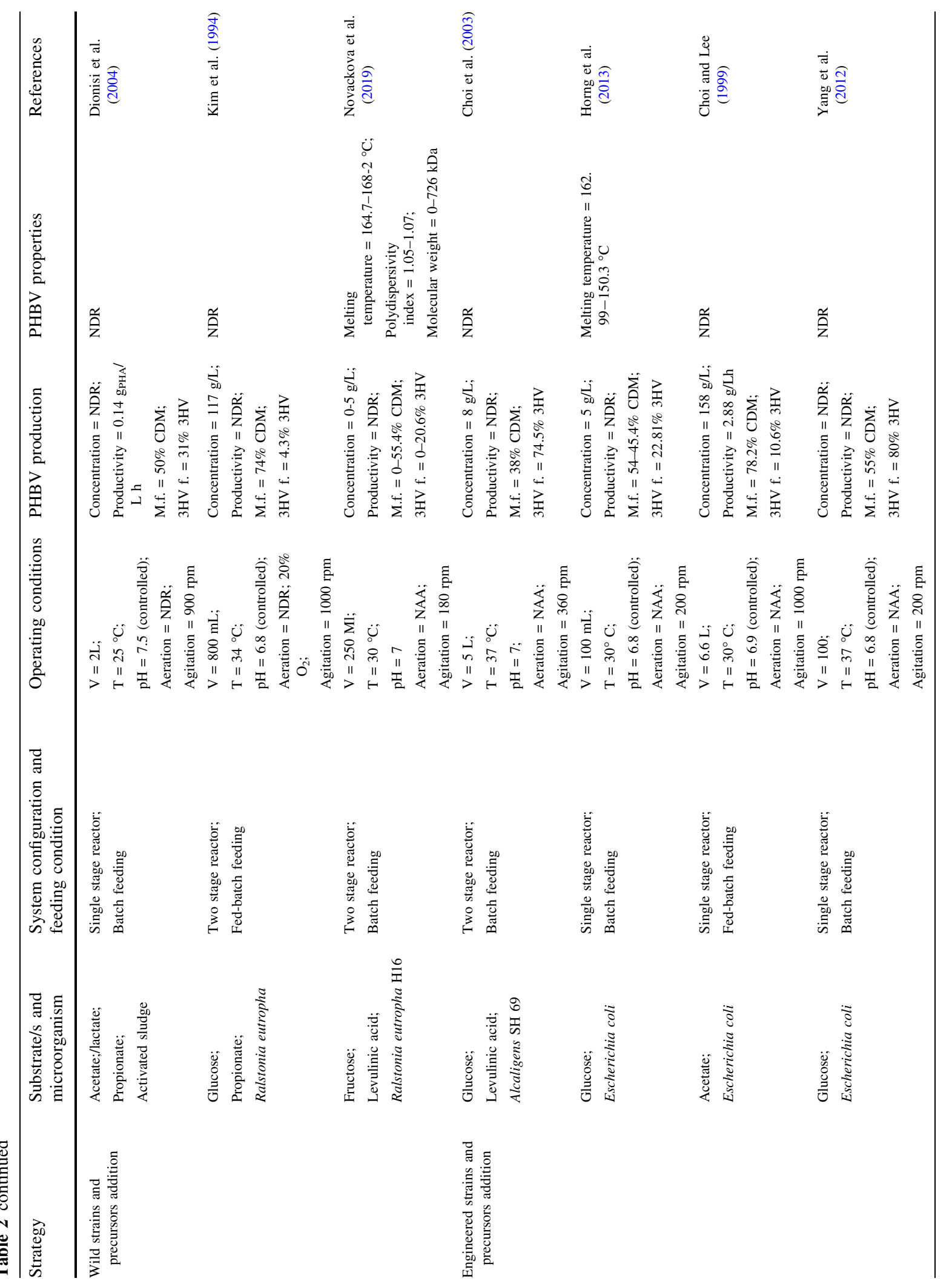




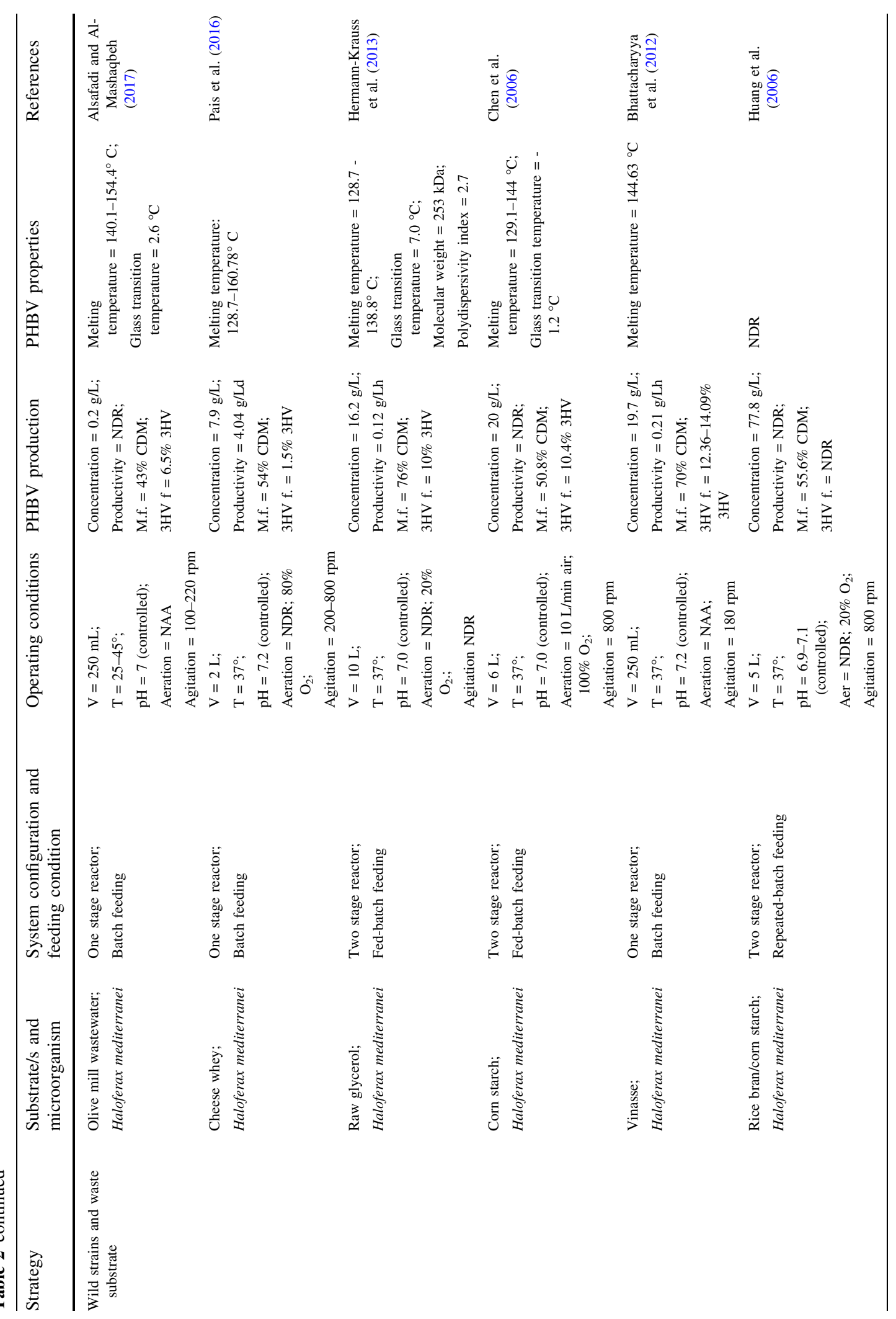




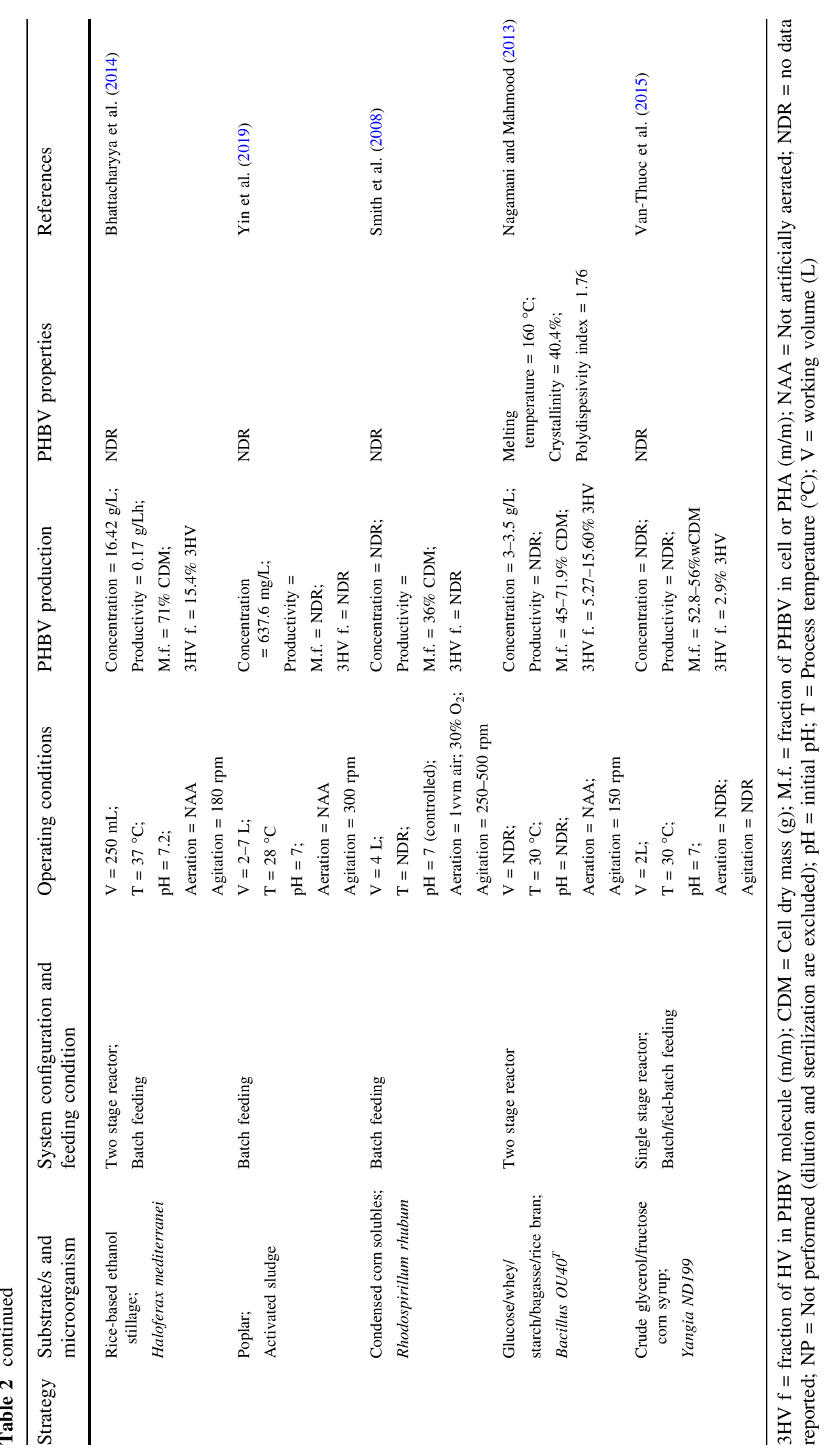


obtained in all the reviewed studies from unrelated carbon sources.

Recently, different studies on open mixed cultures have been conducted with the aim of lowering production costs. Open mixed cultures do not require sterile conditions and are capable to adapt to a very wide variety of complex and inexpensive waste feedstock, thus resulting more interesting in the perspective of a process scale-up.

Arcos-Hernandez et al. (2013) and Dionisi et al. (2004) tested the biomass from activated sludge wastewater treatment plants, obtaining PHBV from a mixture of organic acids by using propionic acid as precursor. Activated sludge was also successfully used to produce PHBV from poplar waste, by Yin et al. (2019). Fergala et al. (2018) investigated the feasibility of enriching methane-utilizing mixed cultures from the anaerobic digestion process: the mixed consortium showed the capability to accumulate PHBV when fed with a mixture of methane and valerate.

In order to avoid sterile conditions, extremophile microorganisms, such as Haloferax Mediterranei can be used. Haloferax Mediterranei belongs to the class of halobacteria that is the extremely halophilic branch of the Archaea domain. This specie tolerates high salinity and requires a concentration of $2-5 \mathrm{M} \mathrm{NaCl}$ for its growth (Alsafadi and Al-Mashaqbeh 2017). The required highly saline environment avoids the growth of other microorganisms in non-sterile conditions, thus significantly reducing process costs. Moreover, Haloferax Mediterranei grows faster compared to the majority of other microorganisms and it is capable to accumulate high amount of PHBV (Koller et al. 2007b). Finally, Haloferax Mediterranei can convert a wide variety of substrates, such as sugars and VFAs (Don et al. 2006; Ferre-Guell and Winterburn 2017, 2019). For such feature, Haloferax Mediterranei has been used for the conversion of different organic waste materials, as follow: whey (Koller et al. 2008; Pais et al. 2016); raw glycerol (Hermann-Krauss et al. 2013); cornstarch (Chen et al. 2006; Huang et al. 2006); vinasse and stillage (Bhattacharyya et al. 2012, 2014); rice bran (Huang et al. 2006); crop waste (Alsafadi et al. 2020).

Due to its high robustness, stability and capacity to degrade complex molecules, Haloferax Mediterranei can produce high PHBV amount even without substrate pre-treatments, thus further reducing process costs, as demonstrated by Bhattacharyya et al. (2014), who used this specie for PHBV production with stillage without any pre-treatment. Similar results were obtained by Hermann-Krauss et al. (2013), who performed only dilution of waste glycerol (10-20 g/ L). Alsafadi \& Al-Mashaqbeh (2017) investigated the conversion of olive mill wastewater (OMW), 5\%, $15 \%, 25 \%, 50 \%, 75 \%$ in volume, respectively, to PHBV by Haloferax Mediterranei. They demonstrated the feasibility of producing PHAs in one-stage cultivation process without the need of pre-treatments, as phenols contained in OMW had no inhibitory effect on the growth of the biomass. An additional advantage of using Haloferax Mediterranei is the absence of need to add precursors for PHBV production.

The major bottleneck in the industrial application of Haloferax Mediterranei is the high quantity of salts required and the production of a high saline effluent, which has to be correctly disposed at the end of the process, even though $96 \%$ of the medium salts can be reused and recovered as reported by Bhattacharyya et al. (2014). Another strategy related to microorganisms is the adoption of metabolic engineered strains, as reported in the following sub-section.

\subsection{Metabolic engineering strategies}

The results achieved in molecular-genetic research and the detailed investigations on the PHBV synthesis have found a convergent point in the study of recombinant strains, which have proven to be efficient in enhancing the production of biopolymers (Koller et al., 2012). Different approaches have been proposed to use metabolic engineering as a strategy to produce and/or increase the accumulation of PHBV.

As previously said, due to the lack of propionyl$\mathrm{CoA}$ in most microorganisms, PHBV production is often hindered by the high costs associated to the supplementation of precursors. Therefore, various studies on metabolic engineering have been conducted with the aim of promoting precursors-independent pathways to produce PHBV. Aldor et al. (2002) engineered the specie Salmonella enterica: the obtained mutant produced propionyl-CoA without the presence of propionate in the culture medium. A recombinant strain of Salmonella enterica serovar Typhimurium (mutant in propionate-activation activity) was metabolically engineered by Aldor and Keasling (2001) to control the composition of the polymer. A gene (prpE) encoding propionyl-CoA 
synthetase was placed under the control of the IPTGinducible taclacUV5 promoter (PtaclacUV5) while the PHA synthesis operon (phaBCA) from Acinetobacter sp. $R A 3849$ was co-expressed under the control of the arabinose-inducible araBAD promoter (PBAD).

Other authors reported the derivation of engineered Escherichia coli strains for PHBV production from unrelated carbon sources. Miscevic et al. (2019) enabled the intracellular formation of non-native propionyl-CoA and investigated various enzymes involved in 3HV biosynthetic pathway from different microorganisms. The engineered Escherichia coli strains produced PHBV from glucose and glycerol as sole carbon sources.

Ma et al. (2018) inserted the phaCAB gene cluster into the bacteria Corynebacterium glutamicum WM001 to enhance the level of intracellular propionyl-CoA. The recombinant strain produced high concentrations of PHBV from glucose with high $3 \mathrm{HV}$ fraction.

Some enzymes converting propionic acid to propionyl-CoA have been used to engineer Escherichia coli strains for PHBV production using propionic acid singularly or combined with other substrates. Usually, mutant strains harbouring other microorganisms genes have been used. For instance, Yang et al. (2012) inserted propionyl-CoA transferase (Pct) into Escherichia coli and produced PHBV containing $>80 \mathrm{wt} \%$ $3 \mathrm{HV}$ content. To promote PHA production in Escherichia coli strains, the authors used the acetoacetyl-CoA reductase (phaB), PHA synthase (phaC) and a b-ketothiolase gene (bktB) from Ralstonia eutropha.

Other authors, instead, investigated strategies for high cell density PHBV production by a recombinant Escherichia coli harbouring the Alcaligens latus PHA biosynthesis genes. The mutant produced a large amount of PHBV with a higher productivity compared to recombinant Escherichia coli harbouring Ralstonia eutropha PHA biosynthesis genes (Choi \& Lee, 1999). Finally, to increase the $3 \mathrm{HV}$ fraction, Horng et al. (2013) cloned the prpE gene encoding propionyl-CoA synthase, the $v g b$ gene encoding bacterial hemoglobin $(\mathrm{VHb})$ and the PHAs synthesis operon (phaCAB) in a plasmid transformed into Escherichia coli XL1-blue. The recombinant specie produced PHBV with increased $3 \mathrm{HV}$ fraction as well as molecular weight.

Another strategy resulting in propionyl-CoA formation has been the induction of the intracellular generation, or over- production, of propionyl-CoA precursors amino acids (e.g. threonine). In the study by Tan et al. (2014), the overexpression of the threonine synthesis pathway and threonine dehydrogenase generated a recombinant Halomonas TDO1 specie capable to produce PHBV using carbohydrates as sole carbon source. Metabolic engineering of the threonine biosynthetic pathway was used by Choi et al. (2003). The authors studied a threonine overproducing mutant of Alcaligenes sp. SH-69 which was capable to produce by using glucose as substrate, an amount of PHBV approximately six folds higher than that achieved by the wild type under the same culture conditions.

The engineering of the tricarboxylic acid (TCA) cycle in Halomonas bluephagenesi has been proposed as well. Chen et al. (2019) performed the study by hampering the $3 \mathrm{HV}$ consumption pathways, thus increasing flux to $3 \mathrm{HV}$ precursor synthesis and activating $\mathrm{ED}$ pathway to reduce $\mathrm{NADH} / \mathrm{NAD}^{+}$ratio for promoting TCA cycle activity via over-expressing bacterial hemoglobin gene vgb. This engineered specie produced PHBV with different $3 \mathrm{HV}$ fractions (0-25 mol\%) from glucose. Shi et al. (2020) proposed a metabolic engineering strategy to produce PHBV from acetate rather than lauric acid with the specie Aeromonas hydrophila. The engineered specie was capable to overexpress b- ketothiolase, acetoacetylCoA reductase, and acetyl-CoA synthetase and it was found capable to produce high PHBV amounts.

A finally strategy to increase the PHBV production has been the conveyance of the substrate conversion towards the PHAs synthesis rather than other metabolites. Zhao et al. (2013) identified a gene cluster involved in EPS biosynthesis in Haloferax mediterranei. Inactivating the genes, they eliminated EPS synthesis. The deficiency in EPS biosynthesis in the mutant strain remarkably increased PHBV accumulation. The productivity of the mutant strain was 20 folds higher when compared to that of the wild strain. To sum up, different types of microorganisms with different peculiarities can be used to enhance the PHBV production process performance. However, the choice of microorganisms cannot be decoupled from the adoption of appropriate substrates. 


\section{Strategies related to substrates}

The microorganism-substrate combination plays an important role in the global process effectiveness. PHBV producing bacteria can utilize a wide variety of organic molecules as substrates, principally sugars, alcohols and organic acids. As previously said, to enhance the process productivity and/or increase the $3 \mathrm{HV}$ fraction, the addition of co-substrates (precursors) is a strategy of main relevance. Many studies investigating the effect of different types of precursors as well as the required precursor dosage in the medium, have been performed, although, as expected, the precursors utilization results in a considerable increase of the production costs. Therefore, the use of waste organic material is reasonably more advisable, as it allows moving the process towards a biorefinery scenario fed with abundant and inexpensive materials (i.e. organic wastes). Moreover, the utilization of pretreatments which generate precursors from wastes has been also proposed as a promising production strategy.

\subsection{Addition of $3 \mathrm{HV}$ fraction precursors}

As mentioned above, the majority of bacteria are capable to produce PHBV instead of PHB only if specific precursors are available. The presence of precursors is also fundamental to adjust the $3 \mathrm{HV}$ monomer fraction in PBHV and, consequently, modify the polymer properties. Therefore, several studies have been addressed to verify the effect of a large number of synthetic carbon sources (e.g. methane, glucose, fructose), linked to these precursors (Table 2). These studies have confirmed that methanotrophic bacteria, Ralstonia eutropha, Pseudomonas species and Hydrogenophaga pseudoflava were capable to produce only PHB when fed solely with the main substrate (Kim et al. 1994; Inn et al. 2003; Myung et al. 2015; Cal et al. 2016; Martla et al. 2018) while the specie Ralstonia eutropha DSM 545 was capable to produce PH3HB4HB when fed with sole glycerol (Cavalheiro et al. 2012). The addition of precursors led, to PHBV production in all cases investigated.

Valeric acid and propionic acid have been the most studied precursors. Valeric acid is clearly a precursor of the $3 \mathrm{HV}$ monomer as it leads to the formation of the 3HV-CoA enzyme, which is successively polymerized (Han et al. 2015). Moreover, valerate concentration in the culture medium strongly affects the $3 \mathrm{HV}$ fraction of the PHBV biopolymer. Myung et al. (2015) tested various combination of $\mathrm{CH}_{4}$ and valerate using a methanotrophic consortium. The authors observed that the $3 \mathrm{HV}$ fraction increased when the valerate concentration as well as the fraction of the oxidized methane were increased. Inn et al. (2003) reached similar results using the bacterium Ralstonia eutropha and butyrate as principal carbon source: a maximum $3 \mathrm{HV}$ fraction of $62 \%$ was reached when a valerate fraction of $100 \%$ was used in the culture medium. Moreover, Sheu et al. (2009) showed that the modification of the valerate concentration in a sugar rich medium could be used to produce the desired $3 \mathrm{HV}$ fraction (from 10 to $90 \%$ ) using the thermophilic bacterium Caldimonas taiwanensis.

The addition of valerate and, therefore, the increase of the $3 \mathrm{HV}$ fraction is relevant as it enhances the quality of the final product. For instance, Koller et al. (2008) observed that the polymer produced through valerate addition presented superior thermal properties compared to the polymer obtained without the precursor addition. According to their analysis, the product quality was appropriate for melt extrusion and film blowing technologies. In the study conducted by Inn et al. (2003), the analysis of the characteristics of the produced polymers showed that an increase of the $3 \mathrm{HV}$ fraction led to a decrease of the melting and glass transition temperatures while the polymer composition did not substantially influence the molecular weight distribution. It is worth noticing that the accumulation of high concentrations of acids in the culture medium can result in bacteria inhibition. To induce the reduction of free protons generation in the cell cytoplasm and avoid the acid accumulation in the medium, Loo and Sudesh (2007) converted the valeric acid into the relative salt prior to feeding cells, thus obtaining a reduced inhibitory effect.

Concerning the use of propionic acid as precursor, it was used in 1970 by Imperial Chemical Industries Ltd. to produce PHBV for the first time. In that case, the 3HV-CoA was obtained from condensation of acetyl-CoA and propionyl-CoA to 3-ketovaleryl-CoA and the subsequent reduction of the condensation product to $3 \mathrm{HV}-\mathrm{CoA}$. These two reactions were catalysed by $\beta$-ketothiolases and acetoacetyl-CoA reductases, respectively (Steinbüchel and Lütke-Eversloh 2003). In the following years, various authors tested different propionate concentrations dissolved in the culture medium. For instance, Yu et al. (2005) used 
culture media containing glucose and three propionate concentrations $(5,7$ and $15 \mathrm{~g} / \mathrm{L})$ with the strain Ralstonia eutropha. The tested media led to increase $3 \mathrm{HV}$ fractions in PBHV (respectively 30\%, 40\% and $60 \%$ ). Similarly, Doi et al. (1987) showed that an increase of the propionate concentration increased the $3 \mathrm{HV}$ fraction in the produced biopolymer (from 22 to 45\%) using the bacteria Ralstonia eutropha H16.

Kim et al. (1994) studied the effect of three different propionic acid to glucose mole ratios $(0.17$, 0.35 , and 0.52) using Ralstonia eutropha NCIMB 11,599. The final PBHV concentrations of 117,74 , and $64 \mathrm{~g} / \mathrm{L}$ with $3 \mathrm{HV}$ fractions of $74 \%, 57 \%$, and $56.5 \%$ respectively, were obtained. Propionate concentration in a glucose medium was also investigated by Park et al. (1997) in presence of Bacillus thuringiensis $\mathrm{R}-510$. The $3 \mathrm{HV}$ fraction increased from 0 to $85 \%$, by increasing propionate concentration from 0 to $0.8 \%$ (w/v). A minimum melting point of $65^{\circ} \mathrm{C}$ was measured when the polymer contained $35 \% 3 \mathrm{HV}$ fraction.

Other authors tested propionic acid addition to culture media and observed that without the addition of the precursor, only PHB was produced. Conversely, the use of propionic acid as co-substrate led to the production of a PHBV polymer with superior thermal and mechanical properties. In particular, the melting temperature, thermal stability, tensile strength and elongation at break were found to be, respectively, $90{ }^{\circ} \mathrm{C}, 220{ }^{\circ} \mathrm{C}, 10.3 \mathrm{MPa}$ and $13.3 \%$ (Balakrishna Pillai et al. 2020). On the other hand, the same side effect responsible for culture inhibition produced by high valerate concentrations occurs with high propionate concentrations. In particular, propionic acid was even found to be more toxic compared to valeric acid. Indeed, Loo and Sudesh (2007) observed that the inhibitory effects of the $3 \mathrm{HV}$ precursors increased in the following order: valerate salt $<$ valeric acid $<$ propionate salt. The formation of Acetyl-Coa from propionic acid was found to be the rate-limiting step in HVCoA formation, thus reducing the substrate consumption rate, when propionate was tested as single substrate. Dionisi et al. (2004) tested lactate, acetate and propionate as single substrates and their mixture. The authors found that when Acetyl-CoA was formed from acetic or lactic acid instead of being formed from sole propionate, higher fractions of the $3 \mathrm{HV}$ monomer were achieved. Moreover, the uptake rate of the propionic acid increased. The importance of using propionic acid as co-substrate rather than as sole carbon source was also underlined by Grousseau et al. (2014). They showed that the simultaneous availability of a second carbon source (butyric acid) led to high conversion rate of propionic acid into $3 \mathrm{HV}$, in presence of Ralstonia eutropha.

Due to the high costs of both valeric and propionic acids, during the last few years, alternative less costly compounds have been tested. For instance, pentanol, which can be oxidized via valeraldehyde to valeric acid and then converted to the $3 \mathrm{HV}$ monomer. It has been demonstrated that increasing pentanol fraction by $20 \%$ in a methanol-pentanol medium resulted in a valerate increase by $50 \%$ and in the stimulation of the PHBV production. Conversely, higher pentanol concentration resulted to be toxic for microorganisms (Ezhov et al. 2013). Despite the reduced costs of the process, pentanol is less effective for PHBV production compared to valerate. Indeed, Cal et al. (2016) tested methanotrophic bacteria fed with sole methane, a mixture of methane with valerate and a mixture of methane with pentanol, by changing the co-substrates concentration. The authors found that the $3 \mathrm{HB} / 3 \mathrm{HV}$ molar ratio in PBHV was directly related to the valerate concentration in the culture medium. The same strain (i.e. Methylocystis WRRC1) produced pure PHB when the process was fed with sole methane and 50\% lower amount of PHBV when it was fed with a mixture of methane with pentanol rather than valerate.

In addition, levulinic acid, the most inexpensive precursor among all those considered, has been tested to increase the $3 \mathrm{HV}$ fraction in PBHV. However, mechanisms leading to PHBV production from levulinic acid have not been clarified yet. Novackova et al. (2019) studied the adaptation of the bacteria Ralstonia eutropha to levulinic acid: the analysis of the PHBV into cells showed a high content of $3 \mathrm{HV}$ when the mentioned precursor was used. The influence of levulinic acid on PHBV production by Ralstonia eutropha was also investigated by Chung et al. (2001): the precursor addition greatly increased the molar fraction of $3 \mathrm{HV}$ from 7 to $75.1 \%$ by increasing the levulinic acid concentration from 0.5 to $4.0 \mathrm{~g} / \mathrm{L}$ in a culture medium containing fructose as main carbon source.

A comparison between the utilization of propionic acid, valeric acid and levulinic acid was performed by Choi et al. (2003). They added precursors to a glucose 
medium using the bacterium Alcaligens SH 69 . Precursors greatly increased the molar fraction of $3 \mathrm{HV}$ to $38-77 \%$. The highest $3 \mathrm{HV}$ fraction of $77 \%$ was reached by adding levulinic acid.

Also, a few studies reported that PHBV could be synthesized through the propionate pathway when some amino acids like threonine, valine and isoleucine act as precursors for propionyl CoA (Steinbüchel and Pieper 1992; Yoon et al. 1995).

Finally, a very interesting and convenient option is neither the use of waste substrates containing precursors or the adoption of waste pre-treatments generating precursors. Indeed, the use of waste and wastewater is a strategy of main relevance for the reduction of process costs.

\subsection{Waste substrates and pre-treatments used to enhance the productivity}

Over the last few years, various organic wastes and wastewaters have been used as feedstock for PHBV production.

One of the most widely used waste has been the crude glycerol, which is the main by-product of biodiesel industry (Cavalheiro et al. 2012; HermannKrauss et al. 2013; Van-Thuoc et al. 2015; Martla et al. 2018). Crude glycerol is particularly suitable for PHAs accumulating species. Indeed, carbon atoms are reduced in glycerol stronger than in any other molecule (e.g. carbohydrates). Consequently, cells using glycerol are in a more evident reduced physiological state, which favours intracellular polymer synthesis (Hermann-Krauss et al. 2013).

It is worth to underline that pure glycerol is actually an expensive material. However, the biodiesel industry residues approximatively $10 \mathrm{~kg}$ of crude glycerol per $100 \mathrm{~L}$ of produced biodiesel. Yield of biodiesel and related by-products are growing annually, thus causing a sharp decrease of crude glycerol cost (Ghosh et al. 2012; Hermann-Krauss et al. 2013).

Van-Thuoc et al. (2015) tested, comparatively, glucose, maltose, xylose, sucrose, fructose, dextrin and glycerol as substrates for PHBV production, thus obtaining the best results with glucose and glycerol in terms of PHA content and with maltose and glycerol in terms of 3HV fraction. Hermann-Krauss et al. (2013) compared the utilization of crude glycerol and pure glycerol to feed Haloferax mediterranei. The authors pointed out that the amount of the polymer produced and its characteristics were almost the same in the two investigated cases. Therefore, due to the abundance of crude glycerol and the limited costs of its production, its use resulted more convenient than that of the pure glycerol.

By-products from the ethanol industry have been tested as well. Smith et al. (2008) used a condensed corn soluble (CCS) medium to feed Rhodospirillum Rhubum. CCS is a coproduct of corn ethanol production and contains organic acids (lactic acid, succinic acid and acetic acid), glycerol, glucose, maltose, dextrins, microelements, phosphorus and a small amount of free nitrogen. Therefore it represents a suitable source of nutrients for different species of bacteria. Bhattacharyya et al. $(2012,2014)$ tested vinasse and stillage, highly polluting wastes of the ethanol industry. Results showed that both substrates were effective for PHBV production, and they could be degraded easily during the process, thus obtaining an important lowering of the organic load at the end of the processes.

Agricultural wastes also represent abundant and inexpensive organic sources. Due to the high carbohydrates content in their hemicellulose and cellulose structures, they can be used for PHBV production.

Chen et al. (2006) showed that corn starch, which is rich in sugars, can be successfully used. Poplar hydrolysate has been positively used as well (Yin et al. 2019), and the use of madhuca flowers from India, which contain a large quantity of sugars, proteins, mineral nutrients and organic acids, has been proposed which success (Anil Kumar et al. 2007; Kerketta and Vasanth 2019).

Among others, rice straw is worldwide the most abundant agricultural waste (approximately 700-800 million tons generated every year). Therefore it can be a potential candidate for the industrial PHA production (Ahn et al. 2016b, a). Nagamani and Mahmood (2013) used rice straw to feed Ralstonia eutropha, thus obtaining better results in terms of PHBV productivity and $3 \mathrm{HV}$ fraction compared to pure glucose, whey, starch and bagasse. Rice bran was compared with wheat bran to replace part of the pure starch in the culture broth by Huang et al. (2006). Both waste substrates increased both the cell concentration and the PHBV accumulation. However, the maximum cell concentration, PHBV concentration and its content were achieved when rice bran was used as co-substrate 
with starch, setting a waste to starch mass ratio of $1: 8$ (w/w).

Due to their high organic load, organic wastes and wastewaters from food industry represent further potentially effective substrates for PHAs accumulating bacteria. A widely utilized waste has been cheese whey, the major by-product from the cheese factories (Nagamani and Mahmood 2013; Pais et al. 2016; Suhazsini et al. 2020). The use of cheese whey for PHBV production in presence of Haloferax mediterranei has revealed to be interesting due to the high salinity requirement of the mentioned bacteria. Indeed, as various types of cheese require along the production process the addition of large quantities of salt, the obtained waste is a high saline cheese whey which already contains the quantity of salt required by Haloferax mediterranei (Pais et al. 2016). Fruit and vegetable wastes represent, certainly, inexpensive and abundant substrates, rich in sugars and nutrients. Vegetable waste has been used as sole carbon source by Ganzeveld et al. (1999). Du \& Yu (2002), instead, coupled anaerobic digestion of food scraps with PHB and PHBV production using the digested food waste as substrate for the PHAs production step. Alsafadi et al. (2020) tested date palm, one of the most successful and vital crops in Middle East region as well as in other arid and semiarid regions, to feed Haloferax mediterranei.

Finally, wastes from vegetable oils production, such as OMW as well as jatropha, sunflower, palm and coconut oils have revealed to be effective for PHBV production (Alsafadi and Al-Mashaqbeh 2017; Lee et al. 2008; Mumtaz et al. 2009; $\mathrm{Ng}$ et al. 2011). Results obtained from the jatropha oil conversion to PHBV revealed that the quality of the produced copolymer was essentially the same as that produced from other pure carbon sources, such as sugars $(\mathrm{Ng}$ et al. 2011).

It has to be highlighted that the use of waste substrates usually requires appropriate pre-treatments, aimed at neither reducing the size and/or the molecular complexity of the organic waste or eliminating toxic compounds.

As it can be noticed from Table 2, physical, chemical or biological pre-treatments can be successfully used. The physical pre-treatment, based on thermal or mechanical processes, are aimed at reducing the solid waste size or extracting simpler molecules. Kerketta \& Vasanth (2019) dried, boiled and filtered madhuca flowers to extract macro and micronutrients. In the study of Alsafadi et al. (2020), both mechanical and thermal pre-treatments were applied to date palm fruit waste: fruit seeds were firstly removed manually, then dates were sliced to small pieces $(1 \mathrm{~cm} \times 1 \mathrm{~cm} \times 0.5 \mathrm{~cm})$. Successively, carbohydrates extraction was performed using a thermal pre-treatment. The thermal extraction was investigated by testing different conditions (e.g. temperature and extraction times). The maximum carbohydrates concentration $(210 \mathrm{~g} / \mathrm{L})$ was obtained using $6 \mathrm{~h}$ extraction time and $40{ }^{\circ} \mathrm{C}$ temperature. Another widely used pre-treatment is the extrusion. Extruders are used to mix and considerably reduce the waste size, in order to facilitate the metabolic activity of bacteria. This treatment was applied to corn starch by Chen et al. (2006) and rice bran by Huang et al. (2006). Both studies compared the utilization of extruded waste and raw waste obtaining better results using the extrudates as carbon source.

Thermal pre-treatments have been also coupled with hydrolysis. Yin et al. (2019) studied hot compressed water method for delignification and promoting the successive enzymatic saccharification of poplar wood. Hot water pre-treatment increased the efficiency of cellulase enzymatic hydrolysis and the yield of reducing sugars. The optimized pre-treatment conditions resulted in being the use of hot water at $200{ }^{\circ} \mathrm{C}$ for $30 \mathrm{~min}$, and the enzymatic hydrolysis at $45^{\circ} \mathrm{C}$ for 3 days. In addition, the conversion of enzymatically hydrolysed cheese whey into PHBV by Haloferax mediterranei was investigated by Martin Koller et al. (2008), while Pais et al. (2016) performed the cheese whey chemical hydrolysis using the same microbial strain. Results obtained using the enzymatic hydrolysis were better in terms of PHBV productivity. However, the study was conducted with precursors addition.

Chemical hydrolysis has been reported to be less expensive than that enzymatic. Moreover, chemical hydrolysis of cheese whey, requiring alkali addition for hydrolysate neutralization, results in a saline substrate, which is an advantage whenever Haloferax mediterranei is utilized as microbial strain. Pais et al. (2016) tested different $\mathrm{HCl}$ concentrations $(0.4,0.7$ and $1.0 \mathrm{M}$ ) and different reaction times (30, 60, $90 \mathrm{~min})$. The most efficient lactose hydrolysis (96\%) with no appreciable degradation of glucose (3.6\%) and 
galactose $(0.9 \%)$ was obtained using $1.0 \mathrm{M} \mathrm{HCl}$ and reaction time of $90 \mathrm{~min}$ (Pais et al. 2016).

Kim et al. (2016) studied the effect of thermal pretreatment and chemical hydrolysis on PHBV production, using rice straw. The pre-treatment conditions strongly affected the substrate composition and the process productivity. The increasing sulfuric acid concentration from 2 to $6 \%$ generated a larger PHBV production while the $3 \mathrm{HV}$ fraction decreased. To obtain a higher $3 \mathrm{HV}$ fraction, an additional heating process of $60 \mathrm{~min}$ was conducted following $2 \%$ sulfuric acid digestion. In such a condition the highest $3 \mathrm{HV}$ mole fraction (22.9\%) was achieved. On the other hand, shorter or longer thermal pre-treatment time resulted in a lower $3 \mathrm{HV}$ fraction. The obtained results were attributed to the generation of both sugars and levulinic acid, which are precursors of the $3 \mathrm{HV}$ fraction.

PHAs production can be coupled with biofuels generation if a biological anaerobic process is conducted as pre-treatment. Such process is inexpensive and environmental-friendly and leads to organic acids generation as soluble products (Pagliano et al. 2017).

Du \& Yu (2002) developed a new technology to couple anaerobic digestion of food scraps with PHBV production. The food wastes were digested in an anaerobic reactor producing acetic, propionic, butyric, and lactic acid. The produced acids were successively transferred through membranes via molecule diffusion into an air-bubbling reactor and utilized to produce PHBV. On the other hand, Mumtaz et al. (2009) used anaerobic fermentation as pre-treatment to obtain a mixture of acetic, butyric and propionic acid, which was successively used for PHBV production by Comamonas.

Bhattacharyya et al. (2012) used, instead, a different process, i.e. adsorption on activate carbon, to pretreat vinasse. This pre-treatment was aimed at removing polyphenolic compounds that are toxic to microorganisms.

Obviously, the convenience of any pre-treatment has to be evaluated by considering the costs/benefits balance. Generally, the use of a substrate rich in simple macro and micro nutrients as well as free of toxic compounds is advisable. For instance, Bhattacharyya et al. (2014) compared the use of stillage with the use of vinasse. The authors reported an increase in the $3 \mathrm{HV}$ fraction using stillage. The improvement was possibly due to the higher amount of available organic acids in stillage, including $3 \mathrm{HV}$ precursors. Moreover, stillage did not require any pre-treatment, while vinasse has to be treated through adsorption, as previously mentioned. Consequently, stillage was more cost effective than vinasse for PHBV production.

\section{Combination of different strategies}

Most of the studies on PHBV production have been conducted using a combination of two or more strategies, as reported in Table 2.

The use of both precursors addition and waste substrates results in a concomitant reduction of process costs and enhancement of the $3 \mathrm{HV}$ fraction. For instance, Sheu et al. (2009) demonstrated that up to $95 \% 3 \mathrm{HV}$ can be accumulated in the produced polymer using food starches and providing a suitable valerate concentration in the culture medium. A further productivity enhancement in terms of polymer concentration/mass fraction can be reached by using the previously mentioned performant wild strains, in addition to precursors utilization and waste substrates. For instance, the combination of plant oils and $3 \mathrm{HV}$ precursors as substrate was evaluated for the biosynthesis of PHBV by Ralstonia eutropa. This combination was suitable for the biosynthesis of high PHBV concentration with high 3HV fraction (Lee et al. 2008; $\mathrm{Ng}$ et al. 2011). Similar results were achieved by Koller et al. (2008), who performed the conversion of whey lactose and valerate to PHBV by Haloferax mediterranei. As previously mentioned, in this case the combination of this specific waste and Haloferax mediterranei further reduced process costs. Indeed, whey contains a high salt concentration, which is required by Haloferax mediterranei. The absence of salt in the waste substrate would have determined an additional cost due to the salt supply. The combination of cheese whey and Haloferax Mediterranei has been tested by Pais et al. (2016), as well, without the addition of precursors. The authors obtained promising results in terms of PHBV production, even though the production was lower compared to that obtained by Koller et al. (2008) by using valerate.

Based on the use of selected strains, in combination with precursors supply, the reduction of costs or the enhancement of the productivity can be reached. For instance, when precursors are used with mixed cultures, such as activated sludge, it is possible to 
enhance the 3HV fraction and avoid sterilization costs (Dionisi et al. 2004). Otherwise, the use of precursors and pure cultures leading to high PHV accumulation (e.g. Ralstonia eutropha) results in the concomitant PHBV accumulation and 3HV fraction enhancement (Kim et al. 1994). Moreover, precursors such as levulinic acid, can be used as a stress factor that favors the selection of microorganisms with desired phenotype. Compared to the parental strain, Ralstonia eutropha species, adapted to levulinic acid, have shown a better growth rate in presence of the mentioned precursor and a higher PHBV accumulation (Novackova et al. 2019).

Another approach concerns the use of precursors in combination with metabolic engineering strains. For instance, Choi et al. (2003) studied the threonine overproducing mutant of Alcaligenes sp. SH-69 using levulinic acid as precursor. The use of metabolic engineering strains resulted in the enhancement of the PHBV accumulation, while levulinic acid enhanced the $3 \mathrm{HV}$ fraction of the produced polymer. Moreover, as previously mentioned, some enzymes converting propionic acid to propionyl-CoA have been used to engineer Escherichia coli strains for PHBV production, using precursors singularly or combined with other substrates (Choi and Lee 1999; Horng et al. 2013; Yang et al. 2012).

Various studies have been conducted by combining the utilization of waste substrates and wild strains which do not require precursors to produce the $3 \mathrm{HV}$ fraction. Combining these two strategies, rather than adding precursors, led to further costs reduction.

In particular, the utilization of waste substrates and pure wild species (i.e. Rhodospirillum rhubum, Bacillus OU40T, Yangia ND199) resulted in the reduction of process costs related to precursors supply (Smith et al. 2008; Nagamani and Mahmood 2013; Van-Thuoc et al. 2015). Moreover, the utilization of waste substrates and mixed consortia (i.e. acivated sludge) avoided sterilization costs, as well (Yin et al. 2019). On the other hand, in this case the production was lower compared to studies performed by using pure cultures. Finally, the utilization of organic wastes in combination with the pure specie Haloferax mediterranei led to high PHBV productions in concomitance with low process costs (Chen et al. 2006; Huang et al. 2006; Bhattacharyya et al. 2012, 2014; HermannKrauss et al. 2013; Pais et al. 2016).

\section{Operating conditions}

In addition to the dependence on the strain and substrate selection, PHBV production processes are strongly affected by other factors such as $\mathrm{pH}$, temperature, aeration conditions, bioreactor design and process regime. Such factors are among the most decisive, especially in the process upscaling. Various bioreactors of different sizes and types have been tested for single stage or multi stage processes under different feeding regimes, extensively reviewed by Koller (2018) and Raza et al. (2019).

The cylindrical stirred tank reactor (STR) apparatus has been used in all studies. This system can be operated discontinuously (batch, repeated batch, fedbatch) or continuously (CSTR).

The batch cultivation mode has been found to be the most used. There are two different approaches developed for batch processes: one-stage cultivation and two-stage cultivation. The choice of the number of stages strongly depends on the selected strain (Koller 2018). It is noteworthy that most bacteria produce PHAs under nutritionally unbalanced conditions. In this case, the biomass growth and PHA accumulation have to be conducted in two different phases. The first one occurs under nutrient-rich conditions and it is characterized by the increase of the microbial biomass concentration. The second one occurs under the running out of an essential nutrient (e.g. nitrogen, phosphorous, sulphur) and it is characterized by an almost constant biomass concentration and an increasing PHAs percentage inside cells. PHAs concentration increases until the external carbon source is depleted (Kourmentza et al. 2017). On the other hand, other strains already can accumulate PHAs under nutritionally balanced conditions. Moreover, PHA-producers with clearly separated phases of biomass and PHAs formation can also contain significant amounts of PHA even without nutrient limitation. In these cases, it is possible to use one stage processes, where cell growth and PHA accumulation occur simultaneously, allowing for costs reduction (Alsafadi and Al-Mashaqbeh 2017).

The batch feeding mode is easy to be operated, but the productions are intrinsically low. Indeed, the maximum concentration of nutrients at the beginning of the process is restricted by the physiological conditions of the used strain (Koller 2018). A simple alternative to the batch cultivation mode is the 
repeated batch mode. It consists in conducting a batch cultivation and then periodically remove a fixed fraction of the fermentation broth that is replaced by the same volume of fresh cultivation medium (Luongo et al. 2019). For instance, Huang et al. (2006) performed a repeated batch fermentation of Haloferax mediterranei on extruded rice bran and corn starch under $\mathrm{pH}$-stat control strategy. The reached high volumetric productivity can be considered an advantage of this strategy over simple batch processes. Moreover, the repeated batch mode saves non-productive time occurring between individual batches.

The same advantage can be obtained using the more common fed-batch mode. In this case substrate is added via substrate pulses when its concentration drops below a critical value, without removing the effluent (Koller 2018). The fed-batch method generally produces higher cell densities compared to the simple batch mode. Consequently, it reduces the overall manufacturing cost and allows for the easier management of the process (Rhee et al., 1993). Both reactor feeding modes have been tested by Ma et al. (2018). The authors achieved higher PHBV production with a higher $3 \mathrm{HV}$ fraction for fed-batch cultures compared to batch ones.

On the other hand, the major drawback of fed-batch cultivation mode is the progressive increase of the fermentation volume, thus causing a concomitant dilution of the fermentation broth (Koller 2018).

Alternative to fed-batch mode is the cell-recycling mode obtained by coupling a membrane module directly with the bioreactor. This type of reactor has been used by Lorantfy et al. (2014), who installed a microfiltration unit for high productive biosynthesis of PHBV. The authors observed a ten-fold increase of volumetric productivity for the fed-

Batch cell recycle mode compared to a continuous chemostat (Lorantfy et al. 2014). Another approach is the continuous fed-batch mode, used by $\mathrm{Du}$ and $\mathrm{Yu}$ (2002). In this case the medium was permanently supplied as a response to the substrate concentration gradient. The authors coupled anaerobic fermentation with PHBV production. The organic acids produced by the acidogenic consortium in the first anaerobic stage were recycled through a tubular membrane module immersed in the fermentation broth of the aerobic reactor. The membrane enabled the permeation of organic acids into the culture broth of the aerobic stage but retained biomass. Using a silicon rubber membrane, only butyrate and acetate passed through, resulting in PHB accumulation. Nevertheless, using a dialysis membrane also enabled the extraction of lactate and propionate, precursors for PHBV production.

Continuous fed-batch processes have been conducted in the case of gaseous substrates as well. For instance, López et al. (2018) coupled anaerobic digestion with PHBV production by the methylotrophic bacterium Methylocystis hirsuta using biogas and VFAs as substrates. Cal et al. (2016) also obtained high $3 \mathrm{HV}$ content using the same reactor configuration with methanotrophic consortia fed with methane and valerate or pentanol. Similarly, purple non sulphur bacteria have been tested in these systems to produce PHBV from CO (Do et al. 2007; Smith et al. 2008).

Other authors conduced continuous processes (CSTR) which, often, are used with the same use of "chemostat" processes ("chemical environment remaining static"). Continuous processes are characterized by steady state conditions as process parameters like concentrations, $\mathrm{pH}$-value, dissolved oxygen tension (pO2), working volume, nutrient supply, etc., are kept constant. The most relevant parameter for the process is the "dilution rate" (D), which is ratio between flow rate (influent and effluent volume per time) and working volume. Too low D values lead to insufficient substrates supply while too high D values lead to the "wash out" condition (Koller 2018). The one-stage chemostat has been used for the first time in PHBV production by Ramsay et al. (1990) using Ralstonia eutropha fed with glucose and propionate and setting a D value of $0.15 \mathrm{~L} / \mathrm{h}$. However, the one stage mode, without the separation of growth and accumulation phases, was not competitive with fedbatch mode setups.

To increase the PHBV accumulation and the substrate consumption, a two- stage process is advisable. For instance, Du and $\mathrm{Yu}$ (2002) selected a drastically higher retention time in the second stage than in the first to boost intracellular PHBV accumulation. Moreover, Ramsay et al. (1990) performed sucrose and propionic acid conversion to PHBV using two-stages process operated at $\mathrm{D}=0.15 \mathrm{~L} / \mathrm{h}$ in both stages. Nitrogen source and propionate were completely utilized in the first stage, while the residual sucrose was used in the second stage to produce an additional PHBV aliquot. 
Generally, in bioreactors, controlled conditions of $\mathrm{pO}_{2}, \mathrm{pH}$-value, and temperature are adopted.

From the analysis of the reviewed studies, it emerged that $96 \%$ of them were conducted under aerobic conditions. To ensure the aerobic environment, reactors have to be equipped with aeration and/ or agitation systems. Usually, air/oxygen rate and agitation speed are adjusted to ensure a dissolved oxygen value of $20-30 \%$ during both the cell growth phase and the PHA accumulation phase. However Vollbrecht and Schlegel (1978) discovered that mutant strains of Ralstonia eutrophus accumulated different PHBV concentrations at different aeration rates. Moreover, Mumtaz et al. (2009) observed that it is possible to obtain high cell dry weight and yield by setting oxygen excess conditions in the growth phase and oxygen-limited conditions during the production phase. Such results are of primary concern in the optimization of process costs, as well. Indeed, aeration has been demonstrated to be significant on computing total costs (Akiyama et al. 2003).

To investigate the optimal oxygen supply for the synthesis of PHBV from organic waste, Ganzeveld et al. (1999) performed a series of experiments varying the airflow during the oxygen limited step. The airflow was varied between 2.4 and $16.8 \mathrm{ml} / \mathrm{min}$. Optimum air flow was found to be $5.1 \mathrm{ml} / \mathrm{min}$. Also, Wagle et al. (2019) lowered the agitation and air inflow rate (rpm from 700 to 350 and vvm from 1 to 0.5 ) and increased the temperature from 28 to $37^{\circ} \mathrm{C}$ to favor PHBV production. The strategy resulted in an appreciable $36 \%$ increase in PHBV production and better substrate utilization.

Other experiments were conducted by adjusting the agitation speed to ensure the oxygen availability.

Cavalheiro et al. (2012), for instance, observed an increase in PHBV accumulation and 3HV fraction when the dissolved oxygen value was set to $2 \%$ rather than $20 \%$ during the growth phase by adjusting agitation and aeration rate. Moreover, Alsafadi and Al-Mashaqbeh (2017) investigated the effect of agitation (100-220 rpm) and found that the best condition for PHBV accumulation was $170 \mathrm{rpm}$.

The growth of microorganisms is influenced by other environmental parameters such as temperature and $\mathrm{pH}$. The temperature influences chemical reactions, metabolism and, consequently, PHBV accumulation. The $\mathrm{pH}$, instead, influences the activity of proteins and enzymes. In almost all studies on PHBV production, the temperature and $\mathrm{pH}$ conditions were set in the ranges of $30-35{ }^{\circ} \mathrm{C}$ and 7-7.5, respectively. However, some authors tested different values to optimize $\mathrm{T}$ and $\mathrm{pH}$ conditions.

For instance, to study the effect of temperature and $\mathrm{pH}$ on PHBV production by Bacillus species, Balakrishna Pillai et al. (2020) varied the incubation temperature between $28^{\circ} \mathrm{C}$ and $40{ }^{\circ} \mathrm{C}$ and the initial $\mathrm{pH}$ between 5 and 9 . The optimum temperature and $\mathrm{pH}$ values for PHBV accumulation were $31^{\circ} \mathrm{C}$ and 7 , respectively. Similarly, optimal values of $30^{\circ} \mathrm{C}$ and pH 7 were observed by Masood et al. (2012) for the specie Bacillus cereus. Moreover, PHBV production by engineered Escherichia coli at $30{ }^{\circ} \mathrm{C}$ was found to be significantly higher than those at $37^{\circ} \mathrm{C}$ (Miscevic et al. 2019).

The only observed exception is the Haloferax Mediterranei specie, which have been found to accumulate higher PHBV amounts at higher temperatures. Indeed, Alsafadi and Al-Mashaqbeh (2017) studied different temperature conditions $\left(25-45^{\circ} \mathrm{C}\right)$ and observed that the optimal value was $37^{\circ} \mathrm{C}$. Moreover, majority of studies on Haloferax mediterranei species have been conducted using the temperature value of $37^{\circ} \mathrm{C}$, thus obtaining good results in terms of PHBV accumulation (Chen et al. 2006; Huang et al. 2006; Bhattacharyya et al. 2012, 2014). (Pais et al. 2016), instead, used the temperature of $45{ }^{\circ} \mathrm{C}$ while Koller et al. (2015) settled the temperature value to $41{ }^{\circ} \mathrm{C}$.

Regarding exceptions in optimal $\mathrm{pH}$ values, the species Halomonas campisalis, examined by Kulkarni et al. (2010) produced PHBV at the value of 9. Moreover, Ganzeveld et al. (1999) found that $\mathrm{pH} 8$, instead of mostly used 7, resulted in faster growth of the bacterium Ralstonia eutrophus. Shimizu et al. (1993) and Vollbrecht and Schlegel (1978) also showed that a $\mathrm{pH}$ value around 8 considerably stimulates PHBV production by the same species. Finally, Loo and Sudesh (2007) varied the initial pH of the medium from 5 to 8 to study optimal values for the bacterium Delftia acidovorans. The highest PHBV accumulation was detected when the $\mathrm{pH}$ was ranging between 7 and 7.5 while the maximum HV fraction was reached when the $\mathrm{pH}$ was set to 5 . However, the effect of the $\mathrm{pH}$ value on PHBV accumulation, was more effective than that observed on the HV fraction. 


\section{Discussion}

As stated several times in this manuscript, PHBV owns superior environmental, thermal and mechanical properties compared to all other bioplastics. However, PHBV high costs and low productivity are still a challenge to be properly faced. Therefore, this review was aimed at reducing the competitiveness gap between PHBV and traditional plastics.

Different strategies for PHBV process productivity enhancement and costs reduction have been analyzed and discussed. In particular, two different approaches have been individuated: the first is related to microorganisms and the second to substrates. Concerning substrates, the discussed strategies have been the addition of precursors of the $3 \mathrm{HV}$ fraction or the use of pre-treated waste materials. Concerning microorganisms, the first discussed strategy has been the use of wild performant species, while the second strategy has been the use of metabolic engineering techniques. Finally, combinations of different strategies and operating conditions have been analyzed. In this section, it is presented a critical discussion on all different strategies. The discussion is conducted with the aim of highlighting the most performing strategies or combination of them to address future researches.

All revised production methods have revealed to be effective to enhance the PHBV productivity and/or reduce costs. In particular, the selection of the most suitable wild microorganisms resulted of primary concern. Among the different species of microorganisms, an interesting option is the selection of bacteria that produce high amounts of PHBV in presence of precursors. Based on results, Ralstonia eutropha is the most performant among others. However, in absence of precursors, this group of bacteria produces exclusively PHB. It is therefore necessary to choose the most convenient precursor to be used as co-substrate. In this case, different suitable possibilities can be considered. In particular, when the addition of synthetic solutions is required, the use of levulinic acid or pentanol is more convenient than other precursors, due to related lower costs. In particular, excellent results in terms of $3 \mathrm{HV}$ fraction enhancement can be obtained by using levulinic acid. Moreover, levulinic acid is competitive with traditional precursors, such as valerate and propionate. However, the addition of synthetic precursors leads to additional costs and increases the difficulties in the process management. Alternatively, it is possible to use waste substrates already containing precursors (e.g. stillage) or to adopt convenient pre-treatments capable to generate precursors (e.g. fermentation).

Another strategy is the use of microorganisms capable to convert unrelated carbon sources (not containing precursors) to PHBV, such as the wild specie Rhodospirillum Rhubum. Moreover, metabolic engineering techniques can be used to promote precursors-independent pathways in a wide range of microorganisms (e.g. Escherichia coli and Salmonella enterica). On the other hand, the limitation of this option is the absence of precursors that does not allow controlling the $3 \mathrm{HV}$ fraction of the produced PHBV. Furthermore, all the mentioned microorganisms are pure species which require a sterile environmental condition.

The requirement of non-sterile conditions in the reaction environment, is crucial for the process scaleup. Consequently, open mixed cultures (e.g. activate sludges), which are capable to adapt to complex unsterile waste substrates, can be used for PHBV production. Alternatively, it can be used Haloferax mediterranei which does not require sterile conditions. This bacterium is one of the most performant specie, due to its high robustness, its stability and its capacity of degrading complex molecules. Moreover, Haloferax Mediterranei can produce high PHBV amount without substrate pre-treatments and without precursors addition. Due to the requirement of a high saline environment, waste substrates containing high salts concentrations such as cheese whey, are preferable. To further enhance the PHBV productivity by Haloferax mediterranei, metabolic engineering techniques can be used to inactivate the gene cluster of the EPS biosynthesis.

The last interesting option to avoid sterilization costs and keep the global process costs down is to focus on a third generation biorefinery approach. In particular, the combined production of energy and materials from waste is gaining great attention. This new approach can replace fossil fuels with organic matter as a source of both biofuels and bioplastics.

For instance, the utilization of anaerobic processes as organic waste pre-treatment for PHBV production processes makes reliable to guarantee precursors availability, and therefore enhance the $3 \mathrm{HV}$ fraction without supplying the process with costly synthetic cosubstrates. Anaerobic cultures can convert organic 
waste to biofuels and a mixture of organic acids, including $3 \mathrm{HV}$ precursors. Performing a double stage anaerobic digestion process can lead to the production of organic acids mixture containing valerate in the first stage and biogas in the second stage. It is possible to recover the produced biogas and use the organic acids mixture for the PHBV production step. Alternatively, both biogas and organic acids can be used in the PHBV production step, when the conversion to PHBV is performed by methanotrophic species.

Also, it is possible to carry out the dark fermentation process in the first anaerobic stage. In this case, it is possible to generate hydrogen and a mixture of organic acids containing propionate. Such a mixture can be successively used for PHBV production in the second aerobic step. Hydrogen recovery from the dark fermentation process represents an important added value. Indeed, hydrogen is the most attractive alternative to fossil fuels due to its high energy content and clean combustion properties.

Finally, the choice of the most suitable substratemicroorganism combination cannot be decoupled from the adoption of appropriate operating conditions, such as reactor configuration and abiotic factors setting. Concerning the reactor configuration, the most effective feeding mode is the continuous fedbatch or the fed-batch system with cell-recycling. In the case of continuous reactors, a two-stage process which separates the growth phase and the accumulation phase promotes a productivity increase. Abiotic factors such as temperature, $\mathrm{pH}$ and agitation have to be settled in order to establish favourable conditions for the microorganisms. Finally, in aerobic processes, oxygen excess conditions in the growth phase followed by oxygen-limited conditions during the production phase result in higher PHBV yields and simultaneously lower production costs.

The mentioned single strategies and their suggested combinations can be successfully studied in future researches on PHBV production. As majority of the reviewed works have been performed on bench scale reactors, it would be worth testing pilot-plants, in order to move forward the process scale-up.

\section{Conclusions}

PHBV is the most promising biopolymer candidate to replace petroleum-based plastics in a wide range of application. However, even though it owns environmental advantages and more suitable properties compared to other bioplastics, the PHBV wide utilization is still limited by its high production costs and low productivity rate. Therefore, the current challenge for researchers is the implementation of efficient and lowcost PHBV production processes. In the present work, based on techno-economic analysis, the authors individuated and presented various production strategies, capable to enhance the PHBV productivity and reduce its costs. The critical evaluation of such strategies outcomes that an appropriate combination of microorganisms with substrates coupled with optimal operating conditions in the framework of the third generation biorefinery approach represents the right direction to enhance the economic competitiveness of PHBV compared to petroleum-based plastics. Therefore, further research on process scale-up could make PHBV the most convenient polymer for the production of totally biodegradable and high performant plastic materials.

Acknowledgements Grazia Policastro would like to thank the Italian Ministry of Education, University and Research (MIUR) who provided financial support for a $\mathrm{PhD}$ fellowship in the frame of the research project entitled "Dipartimenti di Eccellenza" per Ingegneria Civile Edile e AmbientaleCUPE65D18000820006.

Author contributions All the authors equally contributed to this work.

Funding Open access funding provided by Università degli Studi di Napoli Federico II within the CRUI-CARE Agreement. This research did not receive any specific grant from funding agencies in the public, commercial, or not-for-profit sectors.

\section{Declaration}

Conflict of interest The authors declare that they have no conflict of interest.

Open Access This article is licensed under a Creative Commons Attribution 4.0 International License, which permits use, sharing, adaptation, distribution and reproduction in any medium or format, as long as you give appropriate credit to the original author(s) and the source, provide a link to the Creative Commons licence, and indicate if changes were made. The images or other third party material in this article are included in the article's Creative Commons licence, unless indicated otherwise in a credit line to the material. If material is not included in the article's Creative Commons licence and your intended use is not permitted by statutory regulation or exceeds the permitted use, you will need to obtain permission directly 
from the copyright holder. To view a copy of this licence, visit http://creativecommons.org/licenses/by/4.0/.

\section{References}

Ahn J, Jho EH, Kim M, Nam K (2016a) Increased 3HV concentration in the bacterial production of 3-hydroxybutyrate (3HB) and 3-hydroxyvalerate (3HV) copolymer with aciddigested rice straw waste. J Polym Environ 24:98-103. https://doi.org/10.1007/s10924-015-0749-0

Ahn J, Jho EH, Nam K (2016b) Effect of acid-digested rice straw waste feeding methods on the $3 \mathrm{HV}$ fraction of bacterial poly(3-hydroxybutyrate-co-3-hydroxyvalerate) production. Process Biochem 51:2119-2126. https://doi.org/ 10.1016/j.procbio.2016.09.014

Akiyama M, Tsuge T, Doi Y (2003) Environmental life cycle comparison of polyhydroxyalkanoates produced from renewable carbon resources by bacterial fermentation. Polym Degrad Stab 80:183-194. https://doi.org/10.1016/ S0141-3910(02)00400-7

Aldor I, Keasling JD (2001) Metabolic engineering of poly (3hydroxybutyrate-co-3-hydroxyvalerate) composition in recombinant Salmonella enterica serovar typhimurium. Biotech Bioeng 76(2):108-114

Aldor IS, Kim SW, Jones Prather KL, Keasling JD (2002) Metabolic engineering of a novel propionate-independent pathway for the production of poly(3-hydroxybutyrate-co3-hydroxyvalerate) in recombinant Salmonella enterica serovar typhimurium. Appl Environ Microbiol 68:3848-3854. https://doi.org/10.1128/AEM.68.8.38483854.2002

Ali I, Jamil N (2017) Biosynthesis and genetics of polyhydroxyalkanoates by newly isolated Pseudomonas aeruginosa IFS and $30 \mathrm{~N}$ using inexpensive carbon sources. Int $\mathrm{J}$ Environ Sci Technol 14:1879-1888. https://doi.org/10. 1007/s13762-017-1268-4

Alsafadi D, Al-Mashaqbeh O (2017) A one-stage cultivation process for the production of poly-3-(hydroxybutyrate-cohydroxyvalerate) from olive mill wastewater by Haloferax mediterranei. N Biotechnol 34:47-53. https://doi.org/10. 1016/j.nbt.2016.05.003

Alsafadi D, Ibrahim MI, Alamry KA et al (2020) Utilizing the crop waste of date palm fruit to biosynthesize polyhydroxyalkanoate bioplastics with favorable properties. Sci Total Environ 737:139716. https://doi.org/10.1016/j. scitotenv.2020.139716

Anil Kumar PK, Shamala TR, Kshama L et al (2007) Bacterial synthesis of poly(hydroxybutyrate-co-hydroxyvalerate) using carbohydrate-rich mahua (Madhuca sp.) flowers. J Appl Microbiol 103:204-209. https://doi.org/10.1111/j. 1365-2672.2006.03221.x

Arcos-Hernandez MV, Pratt S, Laycock B et al (2013) Waste activated sludge as biomass for production of commercialgrade polyhydroxyalkanoate (PHA). Waste Biomass Valoriz 4:117-127. https://doi.org/10.1007/s12649-0129165-z

Ashori A, Jonoobi M, Ayrilmis N et al (2019) Preparation and characterization of polyhydroxybutyrate-co-valerate (PHBV) as green composites using nano reinforcements.
Int J Biol Macromol 136:1119-1124. https://doi.org/10. 1016/J.IJBIOMAC.2019.06.181

Balakrishna Pillai A, Jaya Kumar A, Kumarapillai H (2020) Biosynthesis of poly(3-hydroxybutyrate-co-3-hydroxyvalerate) (PHBV) in Bacillus aryabhattai and cytotoxicity evaluation of PHBV/poly(ethylene glycol) blends. 3 Biotech 10:1-10. https://doi.org/10.1007/s13205-019-2017-9

Bhattacharyya A, Jana K, Haldar S et al (2015) Integration of poly-3-(hydroxybutyrate-co-hydroxyvalerate) production by Haloferax mediterranei through utilization of stillage from rice-based ethanol manufacture in India and its techno-economic analysis. World J Microbiol Biotechnol 31:717-727. https://doi.org/10.1007/s11274-015-1823-4

Bhattacharyya A, Pramanik A, Maji S et al (2012) Utilization of vinasse for production of poly-3-(hydroxybutyrate-co-hydroxyvalerate) by Haloferax mediterranei. AMB Express 2:34. https://doi.org/10.1186/2191-0855-2-34

Bhattacharyya A, Saha J, Haldar S et al (2014) Production of poly-3-(hydroxybutyrate-co-hydroxyvalerate) by Haloferax mediterranei using rice-based ethanol stillage with simultaneous recovery and re-use of medium salts. Extremophiles 18:463-470. https://doi.org/10.1007/s00792013-0622-9

Bugnicourt E, Cinelli P, Lazzeri A, Alvarez V (2014) Polyhydroxyalkanoate (PHA): review of synthesis, characteristics, processing and potential applications in packaging. Express Polym Lett 8:791-808. https://doi.org/10.3144/ expresspolymlett.2014.82

Byrom D (1992) Production of poly-3-hydroxybutyrate:poly-3hydroxyvalerate copolymers. Microb Rev 103:247-250

Cal AJ, Sikkema WD, Ponce MI et al (2016) Methanotrophic production of polyhydroxybutyrate-co-hydroxyvalerate with high hydroxyvalerate content. Int J Biol Macromol 87:302-307. https://doi.org/10.1016/j.ijbiomac.2016.02. 056

Cavalheiro JMBT, de Almeida MCMD, Grandfils C, da Fonseca MMR (2009) Poly(3-hydroxybutyrate) production by Cupriavidus necator using waste glycerol. Process Biochem 44:509-515. https://doi.org/10.1016/j.procbio.2009. 01.008

Cavalheiro JMBT, Raposo RS, de Almeida MCMD et al (2012) Effect of cultivation parameters on the production of poly(3-hydroxybutyrate-co-4-hydroxybutyrate) and poly(3-hydroxybutyrate-4-hydroxybutyrate-3-hydroxyvalerate) by Cupriavidus necator using waste glycerol. Bioresour Technol 111:391-397. https://doi.org/10.1016/j. biortech.2012.01.176

Chanprateep S (2010) Current trends in biodegradable polyhydroxyalkanoates. J Biosci Bioeng 110:621-632. https://doi. org/10.1016/j.jbiosc.2010.07.014

Chen CW, Don TM, Yen HF (2006) Enzymatic extruded starch as a carbon source for the production of poly(3-hydroxybutyrate-co-3-hydroxyvalerate) by Haloferax mediterranei. Process Biochem 41:2289-2296. https://doi.org/10. 1016/j.procbio.2006.05.026

Chen Y, Chen XY, Du HT et al (2019) Chromosome engineering of the TCA cycle in Halomonas bluephagenesis for production of copolymers of 3-hydroxybutyrate and 3-hydroxyvalerate (PHBV). Metab Eng 54:69-82. https:// doi.org/10.1016/j.ymben.2019.03.006 
Choi J, Lee SY (1997) Process analysis and economic evaluation for poly(3-hydroxybutyrate) production by fermentation. Bioprocess Eng 17:335-342. https://doi.org/10.1007/ s004490050394

Choi J, Lee SY (1999a) High-level production of poly(3-hydroxybutyrate-co-3-hydroxyvalerate) by fed-batch culture of recombinant Escherichia coli. Appl Environ Microbiol 65:4363-4368. https://doi.org/10.1128/aem.65.10.43634368.1999

Choi J, Lee SY (1999b) Factors affecting the economics of polyhydroxyalkanoate production by bacterial fermentation. Appl Microbiol Biotechnol 51:13-21. https://doi.org/ 10.1007/s002530051357

Choi D, Chipman DC, Bents SC, Brown RC (2010) A technoeconomic analysis of polyhydroxyalkanoate and hydrogen production from syngas fermentation of gasified biomass. Appl Biochem Biotechnol 160:1032-1046. https://doi.org/ 10.1007/s12010-009-8560-9

Choi GG, Kim MW, Kim JY, Rhee YH (2003) Production of poly(3-hydroxybutyrate-co-3-hydroxyvalerate) with high molar fractions of 3-hydroxyvalerate by a threonine-overproducing mutant of Alcaligenes sp. SH-69. Biotechnol Lett 25:665-670. https://doi.org/10.1023/A: 1023437013044

Chung SH, Choi GG, Kim HW, Rhee YH (2001) Effect of levulinic acid on the production of poly(3-hydroxybutyrate-co-3-hydroxyvalerate) by Ralstonia eutropha KHB8862. J Microbiol 39:79-82

Dionisi D, Majone M, Papa V, Beccari M (2004) Biodegradable polymers from organic acids by using activated sludge enriched by aerobic periodic feeding. Biotechnol Bioeng 85:569-579. https://doi.org/10.1002/bit.10910

Do YS, Smeenk J, Broer KM et al (2007) Growth of Rhodospirillum rubrum on synthesis gas: conversion of $\mathrm{CO}$ to $\mathrm{H} 2$ and poly- $\beta$-hydroxyalkanoate. Biotechnol Bioeng 97:279-286. https://doi.org/10.1002/bit.21226

Doi Y, Kunioka M, Nakamura Y, Soga K (1987) Biosynthesis of copolyesters in Alcaligenes eutrophus H16 from13C-labeled acetate and propionate. Macromolecules 20:2988-2991. https://doi.org/10.1021/ma00178a006

Don TM, Chen CW, Chan TH (2006) Preparation and characterization of poly(hydroxyalkanoate) from the fermentation of Haloferax mediterranei. J Biomater Sci Polym Ed 17:1425-1438.

https://doi.org/10.1163/ 156856206778937208

Du G, Yu J (2002) Green technology for conversion of food scraps to biodegradable thermoplastic polyhydroxyalkanoates. Environ Sci Technol 36:5511-5516. https://doi. org/10.1021/es011110o

Ezhov VA, Doronina NV, Trotsenko YA (2013) Biosynthesis of polyhydroxybutyrate/valerate with different molecular weights during the growth of Methylobacterium extorquens G-10 on a methanol-pentanol mixture. Appl Biochem Microbiol 49:150-153. https://doi.org/10.1134/ S0003683813020038

Fergala A, Alsayed A, Khattab S et al (2018) Development of methane-utilizing mixed cultures for the production of polyhydroxyalkanoates (PHAs) from anaerobic digester sludge. Environ Sci Technol 52:12376-12387. https://doi. org/10.1021/acs.est.8b04142
Ferre-Guell A, Winterburn J (2017) Production of the copolymer poly(3-hydroxybutyrate-co-3-hydroxyvalerate) with varied composition using different nitrogen sources with Haloferax mediterranei. Extremophiles 21:1037-1047. https://doi.org/10.1007/s00792-017-0964-9

Ferre-Guell A, Winterburn J (2019) Increased production of polyhydroxyalkanoates with controllable composition and consistent material properties by fed-batch fermentation. Biochem Eng J 141:35-42. https://doi.org/10.1016/j.bej. 2018.10.004

Ganzeveld KJ, Van Hagen A, Van Agteren MH et al (1999) Upgrading of organic waste: production of the copolymer poly-3-hydroxybutyrate-co-valerate by Ralstonia eutrophus withorganic waste as sole carbon source. J Clean Prod 7:413-419. https://doi.org/10.1016/s0959-6526(99)001596

Garcia IL, Dorado Perez MP, Lopez JA, Villar MA, Yanniotis S, Koutinas A (2011) Design and techno-economic evaluation of microbial biopolymer production from food industry wastes and agricultural crops. In: ICEF11, international congress on engineering and food, food process engineering in a changing World. Atenas, Greece

Ghosh D, Sobro IF, Hallenbeck PC (2012) Stoichiometric conversion of biodiesel derived crude glycerol to hydrogen: response surface methodology study of the effects of light intensity and crude glycerol and glutamate concentration. Bioresour Technol 106:154-160. https://doi.org/ 10.1016/j.biortech.2011.12.021

Gironi F, Piemonte V (2011) Bioplastics and petroleum-based plastics: strengths and weaknesses. Energy Sources Part A Recover Util Environ Eff 33:1949-1959. https://doi.org/ 10.1080/15567030903436830

Grousseau E, Blanchet E, Déléris S et al (2014) Phosphorus limitation strategy to increase propionic acid flux towards 3-hydroxyvaleric acid monomers in Cupriavidus necator. Bioresour Technol 153:206-215. https://doi.org/10.1016/j. biortech.2013.11.072

Han J, Wu LP, Hou J et al (2015) Biosynthesis, characterization, and hemostasis potential of tailor-made poly(3-hydroxybutyrate-co-3-hydroxyvalerate) produced by Haloferax mediterranei. Biomacromol 16:578-588. https://doi.org/ $10.1021 / \mathrm{bm} 5016267$

Hermann-Krauss C, Koller M, Muhr A et al (2013) Archaeal production of polyhydroxyalkanoate (PHA) co- and terpolyesters from biodiesel industry-derived by-products. Archaea. https://doi.org/10.1155/2013/129268

Horng Y-T, Chien C-C, Huang C-T et al (2013) Biosynthesis of poly(3-hydroxybutyrate-co-3-hydroxyvalerate) with coexpressed propionate permease (prpP), beta-ketothiolase B (bktB), and propionate-CoA synthase (prpE) in Escherichia coli. Biochem Eng J 78:73-79. https://doi.org/10. 1016/j.bej.2012.12.016

Hou J, Feng B, Han J et al (2013) Haloarchaeal-type $\beta$-ketothiolases involved in poly(3-hydroxybutyrate-co-3-hydroxyvalerate) synthesis in Haloferax mediterranei. Appl Environ Microbiol 79:5104-5111. https://doi.org/10.1128/ AEM.01370-13

Huang TY, Duan KJ, Huang SY, Chen CW (2006) Production of polyhydroxyalkanoates from inexpensive extruded rice bran and starch by Haloferax mediterranei. J Ind Microbiol 
Biotechnol 33:701-706. https://doi.org/10.1007/s10295006-0098-z

Inn Z, Eilenmann W, Any $\mathrm{H}$ et al (2003) Tailored synthesis of poly([R]-3-hydroxybutyrate-co-3-hydroxyvalerate) (PHB/ $\mathrm{HV}$ ) in Ralstonia eutropha DSM 428. Acta Biotechnol 23:309-316

Kemavongse K, Prasertsan P, Upaichit A, Methacanon P (2008) Poly- $\beta$-hydroxyalkanoate production by halotolerant Rhodobacter sphaeroides U7. World J Microbiol Biotechnol 24:2073-2085. https://doi.org/10.1007/ s11274-008-9712-8

Kerketta A, Vasanth D (2019) Madhuca indica flower extract as cheaper carbon source for production of poly (3-hydroxybutyrate-co-3-hydroxyvalerate) using Ralstonia eutropha. Process Biochem 87:1-9. https://doi.org/10.1016/j. procbio.2019.09.013

Khanna S, Srivastava AK (2005) Recent advances in microbial polyhydroxyalkanoates. Process Biochem 40:607-619. https://doi.org/10.1016/J.PROCBIO.2004.01.053

Kim BS, Lee SC, Lee SY et al (1994) Production of poly(3hydroxybutyric-co-3-hydroxyvaleric acid) by fed-batch culture of Alcaligenes eutrophus with substrate control using on-line glucose analyzer. Enzyme Microb Technol 16:556-561. https://doi.org/10.1016/01410229(94)90118-X

Koller M (2018) A review on established and emerging fermentation schemes for microbial production of polyhydroxyalkanoate (PHA) biopolyesters. Fermentation. https://doi.org/10.3390/fermentation4020030

Koller M, Atlić A, Gonzalez-Garcia Y et al (2008) Polyhydroxyalkanoate (PHA) biosynthesis from whey lactose. Macromol Symp 272:87-92. https://doi.org/10.1002/masy. 200851212

Koller M, Chiellini E, Braunegg G (2015) Study on the production and re-use of poly(3-hydroxybutyrate-co-3-hydroxyvalerate) and extracellular polysaccharide by the archaeon Haloferax mediterranei strain DSM 1411. Chem Biochem Eng Q 29:87-98. https://doi.org/10.15255/ CABEQ.2014.2058

Koller M, Hesse P, Bona R et al (2007a) Potential of various archae- and eubacterial strains as industrial polyhydroxyalkanoate producers from whey. Macromol Biosci 7:218-226. https://doi.org/10.1002/mabi.200600211

Koller M, Hesse P, Bona R et al (2007b) Biosynthesis of high quality polyhydroxyalkanoate $\mathrm{Co}$ - And terpolyesters for potential medical application by the archaeon haloferax mediterranei. Macromol Symp 253:33-39. https://doi.org/ 10.1002/masy.200750704

Kourmentza C, Plácido J, Venetsaneas N et al (2017) Recent advances and challenges towards sustainable polyhydroxyalkanoate (PHA) production. Bioengineering 4:1-43. https://doi.org/10.3390/bioengineering4020055

Kulkarni SO, Kanekar PP, Nilegaonkar SS et al (2010) Production and characterization of a biodegradable poly(hydroxybutyrate-co-hydroxyvalerate) (PHB-co-PHV) copolymer by moderately haloalkalitolerant Halomonas campisalis MCM B-1027 isolated from Lonar Lake, India. Bioresour Technol 101:9765-9771. https://doi.org/10. 1016/j.biortech.2010.07.089

Laycock B, Halley P, Pratt S et al (2014) The chemomechanical properties of microbial polyhydroxyalkanoates. Prog
Polym Sci 39:397-442. https://doi.org/10.1016/j. progpolymsci.2013.06.008

Lee WH, Loo CY, Nomura CT, Sudesh K (2008) Biosynthesis of polyhydroxyalkanoate copolymers from mixtures of plant oils and 3-hydroxyvalerate precursors. Bioresour Technol 99:6844-6851. https://doi.org/10.1016/j.biortech. 2008.01.051

Li Z, Yang J, Loh XJ (2016) Polyhydroxyalkanoates: opening doors for a sustainable future. NPG Asia Mater 8:1-20. https://doi.org/10.1038/am.2016.48

Liu J, Zhao Y, Diao M et al (2019) Poly(3-hydroxybutyrate-co3-hydroxyvalerate) production by Rhodospirillum rubrum using a two-step culture strategy. J Chem. https://doi.org/ $10.1155 / 2019 / 8369179$

Loo CY, Sudesh K (2007) Biosynthesis and native granule characteristics of poly(3-hydroxybutyrate-co-3-hydroxyvalerate) in Delftia acidovorans. Int J Biol Macromol 40:466-471. https://doi.org/10.1016/j.ijbiomac.2006.11. 003

López JC, Arnáiz E, Merchán L et al (2018) Biogas-based polyhydroxyalkanoates production by Methylocystis hirsuta: a step further in anaerobic digestion biorefineries. Chem Eng J 333:529-536. https://doi.org/10.1016/j.cej. 2017.09.185

Lorantfy B, Ruschitzka P, Herwig C (2014) Investigation of physiological limits and conditions for robust bioprocessing of an extreme halophilic archaeon using external cell retention system. Biochem Eng J 90:140-148. https://doi. org/10.1016/j.bej.2014.06.004

Luongo V, Policastro G, Ghimire A et al (2019) Repeated-batch fermentation of cheese whey for semi-continuous lactic acid production using mixed cultures at uncontrolled $\mathrm{pH}$. Sustainability 11:3330. https://doi.org/10.3390/ su11123330

Ma W, Wang J, Li Y et al (2018) Poly(3-hydroxybutyrate-co-3hydroxyvalerate) co-produced with l-isoleucine in $\mathrm{Co}$ rynebacterium glutamicum WM001. Microb Cell Fact 17:1-12. https://doi.org/10.1186/s12934-018-0942-7

Martla M, Umsakul K, Sudesh K (2018) Production and recovery of poly(3-hydroxybutyrate-co-3-hydroxyvalerate) from biodiesel liquid waste (BLW). J Basic Microbiol 58:977-986. https://doi.org/10.1002/jobm.201800279

Masood F, Hasan F, Ahmed S, Hameed A (2012) Biosynthesis and characterization of poly (3-hydroxybutyrate-co-3-hydroxyvalerate) from Bacillus cereus FA11 isolated from TNT-contaminated soil. Ann Microbiol 62:1377-1384. https://doi.org/10.1007/s13213-011-0386-3

Miscevic D, Srirangan K, Kefale T et al (2019) Heterologous production of 3-hydroxyvalerate in engineered Escherichia coli. Metab Eng. https://doi.org/10.1016/j.ymben.2019.11. 005

Morikawa H, Marchessault RH (1981) Pyrolysis of bacterial polyalkanoates. Can J Chem 59:2306-2313. https://doi. org/10.1139/v81-334

Mumtaz T, Abd-Aziz S, Rahman NA et al (2009) Fed-batch production of $\mathrm{P}(3 \mathrm{HB}-\mathrm{co}-3 \mathrm{HV})$ copolymer by Comamonas sp EB 172 using mixed organic acids under dual nutrient limitation. Eur J Sci Res 33:374-384

Myung J, Galega WM, Van Nostrand JD et al (2015) Long-term cultivation of a stable Methylocystis-dominated methanotrophic enrichment enabling tailored production of 
poly(3-hydroxybutyrate-co-3-hydroxyvalerate). Bioresour Technol 198:811-818. https://doi.org/10.1016/j.biortech. 2015.09.094

Nagamani P, Mahmood S (2013) ISSN 6299 production of poly(3-hydroxybutyrate-co-3-hydroxyvalerate ) by a novel bacillus OU40 t from inexpensive carbon sources. Int $\mathbf{J}$ Pharma Biosci 4:182-193

Ng KS, Wong YM, Tsuge T, Sudesh K (2011) Biosynthesis and characterization of poly(3-hydroxybutyrate-co-3-hydroxyvalerate) and poly(3-hydroxybutyrate-co-3-hydroxyhexanoate) copolymers using jatropha oil as the main carbon source. Process Biochem 46:1572-1578. https://doi.org/ 10.1016/j.procbio.2011.04.012

Novackova I, Kucera D, Porizka J et al (2019) Adaptation of Cupriavidus necator to levulinic acid for enhanced production of P(3HB-co-3HV) copolyesters. Biochem Eng J 151:107350. https://doi.org/10.1016/j.bej.2019.107350

Pagliano G, Ventorino V, Panico A, Pepe O (2017) Integrated systems for biopolymers and bioenergy production from organic waste and by-products: a review of microbial processes. Biotechnol Biofuels 10:1-24. https://doi.org/10. 1186/s13068-017-0802-4

Pais J, Serafim LS, Freitas F, Reis MAM (2016) Conversion of cheese whey into poly(3-hydroxybutyrate-co-3-hydroxyvalerate) by Haloferax mediterranei. New Biotechnol 33:224-230. https://doi.org/10.1016/j.nbt.2015.06.001

Park SK, Lee KT, Kim YB, Rhee YH (1997) Biosynthesis of polyhydroxybutyrate and of poly-(3hydroxybutyrate-co3hydroxyvalerate) by Bacillus thuringiensis R-510. J Microbiol 35:127-133

Pérez-Pantoja D, De La Iglesia R, Pieper DH, González B (2008) Metabolic reconstruction of aromatic compounds degradation from the genome of the amazing pollutantdegrading bacterium Cupriavidus necator JMP134

Phukon P, Saikia JP, Konwar BK (2012) Bio-plastic (P-3HB-co3HV) from Bacillus circulans (MTCC 8167) and its biodegradation. Colloids Surf B Biointerfaces 92:30-34. https://doi.org/10.1016/j.colsurfb.2011.11.011

Policastro G, Luongo V, Fabbricino M (2020) Biohydrogen and poly- $\beta$-hydroxybutyrate production by winery wastewater photofermentation: effect of substrate concentration and nitrogen source. J Environ Manag 271:111006. https://doi. org/10.1016/j.jenvman.2020.111006

Poltronieri P, Kumar P (2017) Polyhydroxyalcanoates (PHAs) in industrial applications BT. In: Martínez LMT, Kharissova OV, Kharisov BI (eds) Handbook of ecomaterials. Springer, Cham, pp 1-30

Ramsay BA, Lomaliza K, Chavarie C et al (1990) Production of poly-(p-hydroxybutyric-co-3-hydroxyvaleric) acids. Appl Environ Microbiol 56:2093-2098

Raza ZA, Tariq MR, Majeed MI, Banat IM (2019) Recent developments in bioreactor scale production of bacterial polyhydroxyalkanoates. Bioprocess Biosyst Eng 42:901-919. https://doi.org/10.1007/s00449-019-02093-x

Rivera-Briso AL, Serrano-Aroca Á (2018) Poly(3-hydroxybutyrate-co-3-hydroxyvalerate): enhancement strategies for advanced applications. Polymers (Basel) 10:1-28. https:// doi.org/10.3390/polym10070732

Sheu DS, Chen WM, Yang JY, Chang RC (2009) Thermophilic bacterium Caldimonas taiwanensis produces poly(3-hydroxybutyrate-co-3-hydroxyvalerate) from starch and valerate as carbon sources. Enzyme Microb Technol 44:289-294. https://doi.org/10.1016/j.enzmictec.2009.01. 004

Shi LL, Da YY, Zheng WT et al (2020) Production of polyhydroxyalkanoate from acetate by metabolically engineered Aeromonas hydrophilia. J Biosci Bioeng. https://doi.org/ 10.1016/j.jbiosc.2020.05.003

Shimizu H, Tamura S, Shioya S, Suga K (1993) Kinetic study of poly-d(-)-3-hydroxybutyric acid (PHB) production and its molecular weight distribution control in a fed-batch culture of Alcaligenes eutrophus. J Ferment Bioeng 76:465-469. https://doi.org/10.1016/0922-338X(93)90242-Z

Smith RL, West TP, Gibbons WR (2008) Rhodospirillum rubrum: Utilization of condensed corn solubles for poly-(3hydroxybutyrate-co-3-hydroxyvalerate) production. J Appl Microbiol 104:1488-1494. https://doi.org/10.1111/ j.1365-2672.2007.03685.x

Steinbüchel A, Lütke-Eversloh T (2003) Metabolic engineering and pathway construction for biotechnological production of relevant polyhydroxyalkanoates in microorganisms. Biochem Eng J 16:81-96. https://doi.org/10.1016/S1369703X(03)00036-6

Steinbüchel A, Pieper U (1992) Production of a copolyester of 3-hydroxybutyric acid and 3-hydroxyvaleric acid from single unrelated carbon sources by a mutant of Alcaligenes eutrophus. Appl Microbiol Biotechnol 37:1-6. https://doi. org/10.1007/BF00174193

Strong P, Laycock B, Mahamud S et al (2016) The Opportunity For High-Performance Biomaterials From Methane. Microorganisms 4:11. https://doi.org/10.3390/ microorganisms4010011

Suhazsini P, Keshav R, Narayanan S et al (2020) A Study on the synthesis of poly (3-hydroxybutyrate-co-3-hydroxyvalerate) by Bacillus megaterium utilizing cheese whey permeate. J Polym Environ 28:1390-1405. https://doi.org/10. 1007/s10924-020-01687-x

Tan D, Wu Q, Chen JC, Chen GQ (2014) Engineering halomonas TD01 for the low-cost production of polyhydroxyalkanoates. Metab Eng 26:34-47. https://doi.org/10.1016/ j.ymben.2014.09.001

Tebaldi ML, Maia ALC, Poletto F et al (2019) Poly(-3-hydroxybutyrate-co-3-hydroxyvalerate) (PHBV): current advances in synthesis methodologies, antitumor applications and biocompatibility. J Drug Deliv Sci Technol 51:115-126. https://doi.org/10.1016/j.jddst.2019.02.007

Ueda S, Matsumoto S, Takagi A, Yamane T (1992) Synthesis of poly(3-hydroxybutyrate-co-3-hydroxyvalerate) from methanol and n-amyl alcohol by the methylotrophic bacteria Paracoccus denitrificans and Methylobacterium extorquens. Appl Environ Microbiol 58:3574-3579. https://doi.org/10.1128/aem.58.11.3574-3579.1992

Van-Thuoc D, Huu-Phong T, Minh-Khuong D, Hatti-Kaul R (2015) Poly(3-hydroxybutyrate-co-3-hydroxyvalerate) production by a moderate halophile Yangia sp. ND199 using glycerol as a carbon source. Appl Biochem Biotechnol 175:3120-3132. https://doi.org/10.1007/ s12010-015-1479-4

Verlinden RAJ, Hill DJ, Kenward MA et al (2011) Production of polyhydroxyalkanoates from waste frying oil by cupriavidus necator. AMB Express 1:1-8. https://doi.org/10. 1186/2191-0855-1-11 
Vollbrecht D, Schlegel HG (1978) Excretion of metabolites by hydrogen bacteria II. Influences of aeration, $\mathrm{pH}$, temperature, and age of cells. Eur J Appl Microbiol Biotechnol 6:157-166. https://doi.org/10.1007/BF00504427

Vu DH, Åkesson D, Taherzadeh MJ, Ferreira JA (2020) Recycling strategies for polyhydroxyalkanoate-based waste materials: an overview. Bioresour Technol. https://doi.org/ 10.1016/j.biortech.2019.122393

Wagle AR, Dixit YM, Vakil BV (2019) Scale up studies for polyhydroxyalkanoate production by a Bacillus flexus strain with industrial potential. Indian $\mathrm{J}$ Microbiol 59:383-386. https://doi.org/10.1007/s12088-019-00807-z

Wegen RJVAN, Ling Y, Member APJM (1998) Polyhydroxyalkanoates using Escherichia coli: an economic analysis. Chem Eng Res Des 76:417-426

Williams DR, Anderson AJ, Dawes EA, Ewing DF (1994) Production of a co-polyester of 3-hydroxybutyric acid and 3-hydroxyvaleric acid from succinic acid by Rhodococcus ruber: biosynthetic considerations. Appl Microbiol Biotechnol 40:717-723. https://doi.org/10.1007/ BF00173334

Wu SC, Liou SZ, Lee CM (2012) Correlation between biohydrogen production and polyhydroxybutyrate (PHB) synthesis by Rhodopseudomonas palustris WP3-5. Bioresour Technol 113:44-50. https://doi.org/10.1016/J. BIORTECH.2012.01.090

Yang YH, Brigham CJ, Song E et al (2012) Biosynthesis of poly(3-hydroxybutyrate-co-3-hydroxyvalerate) containing a predominant amount of 3-hydroxyvalerate by engineered Escherichia coli expressing propionate-CoA transferase. J Appl Microbiol 113:815-823. https://doi.org/10.1111/j. 1365-2672.2012.05391.x

Yin F, Li D, Ma X, Zhang C (2019) Pretreatment of lignocellulosic feedstock to produce fermentable sugars for poly(3hydroxybutyrate-co-3-hydroxyvalerate) production using activated sludge. Bioresour Technol. https://doi.org/10. 1016/j.biortech.2019.121773

Yoon JS, Kim JY, Rhee YH (1995) Effects of amino acid addition on molar fraction of 3-hydroxyvalerate in copolyester of 3-hydroxybutyrate and 3-hydroxyvalerate synthesized by Alcaligenes sp. SH-69. J Ferment Bioeng 80:350-354. https://doi.org/10.1016/0922$338 X(95) 94203-4$

Yu L, Dean K, Li L (2006) Polymer blends and composites from renewable resources. Prog Polym Sci 31:576-602. https:// doi.org/10.1016/J.PROGPOLYMSCI.2006.03.002

Yu ST, Lin CC, Too JR (2005) PHBV production by Ralstonia eutropha in a continuous stirred tank reactor. Process Biochem 40:2729-2734. https://doi.org/10.1016/j.procbio. 2004.12.023

Zakaria MR, Ariffin H, Abd-Aziz S et al (2013) Improved properties of poly(3-hydroxybutyrate-co-3-hydroxyvalerate) produced by Comamonas sp EB172 utilizing volatile fatty acids by regulating the nitrogen source. Biomed Res Int. https://doi.org/10.1155/2013/237806

Zembouai I, Bruzaud S, Kaci M et al (2014) Mechanical recycling of poly(3-hydroxybutyrate-co-3-hydroxyvalerate)/ polylactide based blends. J Polym Environ 22:449-459. https://doi.org/10.1007/s10924-014-0684-5

Zhao D, Cai L, Wu J et al (2013) Improving polyhydroxyalkanoate production by knocking out the genes involved in exopolysaccharide biosynthesis in Haloferax mediterranei. Appl Microbiol Biotechnol 97:3027-3036. https://doi.org/ 10.1007/s00253-012-4415-3

Zheng Y, Chen J-C, Ma Y-M, Chen G-Q (2020) Engineering biosynthesis of polyhydroxyalkanoates (PHA) for diversity and cost reduction. Metab Eng 58:82-93. https://doi.org/ 10.1016/J.YMBEN.2019.07.004

Zuñiga C, Morales M, Revah S (2013) Polyhydroxyalkanoates accumulation by Methylobacterium organophilum CZ-2 during methane degradation using citrate or propionate as cosubstrates. Bioresour Technol 129:686-689. https://doi. org/10.1016/j.biortech.2012.11.120

Publisher's Note Springer Nature remains neutral with regard to jurisdictional claims in published maps and institutional affiliations. 